\title{
Norois
}

Environnement, aménagement, société

\section{Le plateau littoral du Bas-Léon (nord Finistère, Bretagne) au Ier millénaire avant notre ère : perspectives pour une lecture croisée sur les dynamiques du peuplement et du paysage}

The coastal plateau of Lower Léon (nord Finistère, Brittany) during the 1st millennium BC: perspectives for crossing approaches of the peopling and landscape dynamics

Marie-Yvane Daire, Dominique Marguerie, Muriel Fily, Anna Baudry, Laurent Quesnel et Tristan Arbousse-Bastide

\section{(2) OpenEdition \\ Journals}

Édition électronique

URL : http://journals.openedition.org/norois/3685

DOI : 10.4000/norois.3685

ISSN : $1760-8546$

Éditeur

Presses universitaires de Rennes

Édition imprimée

Date de publication : 30 novembre 2011

Pagination : 95-119

ISBN : 978-2-7535-1765-3

ISSN : 0029-182X

Référence électronique

Marie-Yvane Daire, Dominique Marguerie, Muriel Fily, Anna Baudry, Laurent Quesnel et Tristan Arbousse-Bastide, "Le plateau littoral du Bas-Léon (nord Finistère, Bretagne) au ler millénaire avant notre ère : perspectives pour une lecture croisée sur les dynamiques du peuplement et du paysage », Norois [En ligne], 220 | 2011, mis en ligne le 30 novembre 2013, consulté le 02 mai 2019. URL : http:// journals.openedition.org/norois/3685; DOI : 10.4000/norois.3685

Ce document a été généré automatiquement le 2 mai 2019.

(c) Tous droits réservés 


\title{
Le plateau littoral du Bas-Léon (nord Finistère, Bretagne) au Ier millénaire avant notre ère : perspectives pour une lecture croisée sur les dynamiques du peuplement et du paysage
}

\author{
The coastal plateau of Lower Léon (nord Finistère, Brittany) during the 1st \\ millennium BC: perspectives for crossing approaches of the peopling and \\ landscape dynamics
}

Marie-Yvane Daire, Dominique Marguerie, Muriel Fily, Anna Baudry, Laurent Quesnel et Tristan Arbousse-Bastide

\footnotetext{
Dans une démarche relevant plus de «l'archéogéographie » que de la géoarchéologie (selon la distinction proposée par G. Chouquer, 2003), cette approche consiste ici à nous interroger sur les dynamiques spatio-temporelles des milieux côtiers mais aussi des groupes humains sur un pas de temps couvrant le dernier millénaire avant notre ère. Le littoral constitue un environnement riche de la variété de ses milieux et de ses ressources, ce qui illustre assez bien la formule «the power of aquatic perimeters" (Fitzpatrick et Anderson, 2008). Environnement complexe, le milieu littoral apparaît en perpétuelle construction quand on tente d'analyser son évolution passée, évolution dont les marqueurs sont plus ou moins évidents selon les secteurs géographiques et selon les sites. Riche de la diversité de ses faciès géomorphologiques (côtes rocheuses plus ou moins basses, rias/abers, larges estrans sableux, dunes), ce paysage est perpétuellement façonné par des agents dynamiques tels que les variations du niveau marin, la houle, le vent, les marées et les flux de sédiments, autant de facteurs qui modèlent un trait de côte en continuelle mouvance.
} 
2 Le cadre géographique de cette étude est centré sur la portion de la frange littorale du Bas-Léon comprise entre la baie de Goulien au nord-est et la commune de Plouarzel, au sud-ouest, soit le "pays des abers" dans le Finistère (fig. 1). Terre de contrastes, ce territoire se présente comme un plateau de faible altitude dont la portion sud offre un paysage à falaises correspondant au rebord du plateau, tandis que l'ouest et le nord sont caractérisés par des côtes basses et sablonneuses, entaillées par les profondes échancrures des abers. Vers le large, cette côte est bordée par la " plate-forme à écueils ", large de 1 à $5 \mathrm{~km}$, qui porte les îles et les rochers de la côte, étendue riche de champs d'algues abritant une faune abondante et diversifiée (Hallégouët, 1971; Brigand, 2002 ; Elégoët, 2007).

3 L'espace considéré prodigue, outre des milieux variés, des témoignages d'occupations humaines de toutes époques, depuis le Paléolithique, mais qui ne s'inscrivent d'ailleurs pas obligatoirement dans une continuité spatio-temporelle. Sur le plan chronologique, notre approche sera limitée au Ier millénaire av. n.è. : du point de vue culturel, ce pas de temps est centré sur la période de l'Âge du Fer (800-50 av. n.è.) et inclut en amont l'Âge du Bronze final dit «Atlantique » et, en aval, les premières décennies de l'époque galloromaine. Ce $\mathrm{I}^{\mathrm{er}}$ millénaire intègre des phases de transitions culturelles dont il convient d'analyser les rythmes et la dynamique : d'une part, le passage de l'Âge du Bronze à l'Âge du Fer, qui ne semble pas correspondre seulement à une évolution de la métallurgie mais à un lent et progressif bouleversement socio-économique ; d'autre part, un changement là encore progressif, mais assez radical, de la société, consécutif à la conquête romaine, mais dont les rythmes semblent différer selon les contextes. Des transformations, des évolutions vont donc se faire jour, non seulement dans le domaine de la culture matérielle (pratiques architecturales, circuits d'échanges de biens et de denrées, évolutions technologiques...) mais aussi dans les activités économiques voire dans la société et donc dans les relations entre l'homme et le milieu naturel (Giot et al., 1995).

D'un point de vue environnemental, ce pas de temps voit une évolution des paysages, conditionnée par des contraintes naturelles (évolution du trait de côte, érosion du littoral, etc.) ainsi que par des forçages anthropiques divers.

5 Le plateau littoral du Bas-Léon a été retenu ici en tant que cadre géographique, pour la densité et la diversité des données, tant culturelles que paysagères. Une analyse critique des données nous amènera cependant à souligner certaines limites de cette documentation.

6 Ce secteur apparaît aujourd'hui comme une zone très documentée où il sera possible de vérifier la fertilité scientifique d'une démarche consistant à croiser les regards d'archéologues et d'environnementalistes pour répondre à des problématiques liées aux interactions homme/milieu : dans quelle mesure les ressources environnementales ontelles conditionné les implantations humaines? Dans quelle mesure l'évolution du peuplement trahit-elle des adaptations $\mathrm{au}(\mathrm{x})$ milieu(x) naturel(s)? Quels sont les marqueurs de l'impact des activités anthropiques sur l'environnement?

7 Les limites de cette démarche et du présent article tiennent cependant à l'état actuel de la recherche dans cette micro-région, avec une hétérogénéité des données, à la fois sur le plan chronologique et sur les plans disciplinaires. Si certains sites, de l'Âge du Fer en particulier, ont été bien documentés $d u$ point de vue des analyses paléoenvironnementales, ces données ne peuvent fournir que des points de référence qu'il est toujours délicat d'extrapoler. Simultanément, les données archéologiques sont 
très inégales, dans la mesure où la majorité des sites ne sont connus que par prospection ou inventaire, avec des degrés d'imprécision inhérents aux types de vestiges considérés.

Les cartographies proposées ici (fig. 1), mettant en regard les données pour l'ensemble de l'Âge du Bronze (de 2200 à 800 av. n.è. environ) (fig. 1B) et pour l'Âge du Fer (de 800 à 50 av. n.è. environ) (fig. 1C) n'ont donc qu'une valeur documentaire assez générale. Elles ont cependant le mérite de montrer la densité de l'information archéologique disponible et des tendances très générales mais ne sauraient toutefois être utilisées comme supports d'une analyse spatiale fine du peuplement protohistorique dans cette zone géographique. En l'état actuel de la recherche, des tentatives de ce genre ne pourront se faire que sur des secteurs géographiques bien circonscrits et particulièrement bien documentés, comme cela a été réalisé pour l'archipel de Molène (Pailler et al., ce volume), et comme cela sera tenté infra pour le secteur de l'Aber Ildut.

Par ailleurs, nous évoquerons dans cette étude certaines structures dont la datation n'est pour l'heure pas établie de manière formelle, mais qui participent à la réflexion méthodologique au cas par cas, lorsqu'un contexte environnant peut-être exploité à une échelle locale : il s'agit, d'une part, d'enclos détectés d'avion, et, d'autre part, de pêcheries ou barrages à poissons généralement localisés dans la bande intertidale.

10 Malgré les limites évoquées, cette démarche débouche sur une synthèse inédite du peuplement du Bas-Léon et de l'environnement au cours des Âges des Métaux, complétant ainsi les connaissances des périodes antérieures, et du Néolithique en particulier, pour lesquelles un panorama d'ensemble a déjà été proposé (Sparfel et Pailler, 2009).

11 Ces données seront traitées selon le schéma analytique suivant: dans un premier temps, les données archéologiques et paléoenvironnementales seront présentées puis exploitées afin de dégager des indicateurs de l'évolution du paysage côtier dans le Bas-Léon tout en soulignant les limites d'une analyse de l'évolution des peuplements ; cette dernière nous conduira à développer notamment le cas des sites archéologiques de l'île d'Yoc'h et de l'île Guennoc, particulièrement bien documentés. Les dynamiques du peuplement et du paysage seront ensuite illustrées sous deux angles complémentaires, à savoir un peuplement conditionné par les contraintes environnementales mais aussi un paysage largement façonné par l'homme et le poids respectif de ces divers processus fera ensuite l'objet d'une discussion.

\section{Les données archéologiques et paléoenvironnementales}

\section{Les indicateurs de l'évolution du paysage côtier du Bas-Léon}

12 À l'extrémité nord-ouest de la péninsule armoricaine, le Léon est baigné à l'ouest par l'océan Atlantique et, au nord par la Manche; les côtes rocheuses, découpées mais sans haute falaise alternent avec quelques beaux estrans sableux, ceinturés par des cordons dunaires. Le littoral du Bas-Léon est entaillé par de longues et profondes rias, les abers Ildut, Wrac'h et Benoit, par lesquelles la mer peut pénétrer profondément dans les terres à la faveur des marées hautes; ces rias constituent autant de havres pour les navigateurs, mais aussi des axes de pénétration vers l'intérieur des terres, tandis qu'ils délimitent des entités ou unités géographiques en compliquant la circulation côtière par voie terrestre. 
Ce territoire $\mathrm{du}$ Léon possède en outre un réseau hydrographique au maillage très serré (Hallégouët, 1971).

Le $\mathrm{I}^{\text {er }}$ millénaire av. n.è. est marqué par une évolution climatique et des transformations du paysage, notamment le trait de côte qui est soumis à des variations parfois importantes (Giot, 1990). Au cours de l'Âge du Bronze, entre 3800 et 3000 B.P., des dépôts littoraux organiques du Léon montrent des influences saumâtres à des niveaux marins voisins des plus hautes mers actuelles, comme si cet intervalle de temps avait connu un épisode transgressif correspondant au Bronze moyen. Une régression apparente de 2 à $3 \mathrm{~m}$ se serait produite entre 3000 et 2700 B.P., favorisant l'exondation de vastes surfaces sableuses et la mise en place de grands massifs dunaires (Hallégouët, 1981; Guilcher et Hallégouët, 1991) ; cette régression de l'Âge du Bronze pose cependant un certain nombre de questions et son existence est à nuancer au vu des résultats des recherches les plus récentes (Stéphan, 2008 ; Stéphan et al., 2009, ce volume).

Figure 1 : A - Localisation de la zone d'étude ; B - Distribution des sites de l'Âge du Bronze sur le territoire pris en compte ; $C$ - Distribution des sites de l'Âge du Fer sur le territoire pris en compte (DAO L. Quesnel)/A - Location map of the study area; B - Distribution of the Bronze Age sites on the considered area; C - Distribution of the Bronze Age sites on the considered area (DAO L. Quesnel)

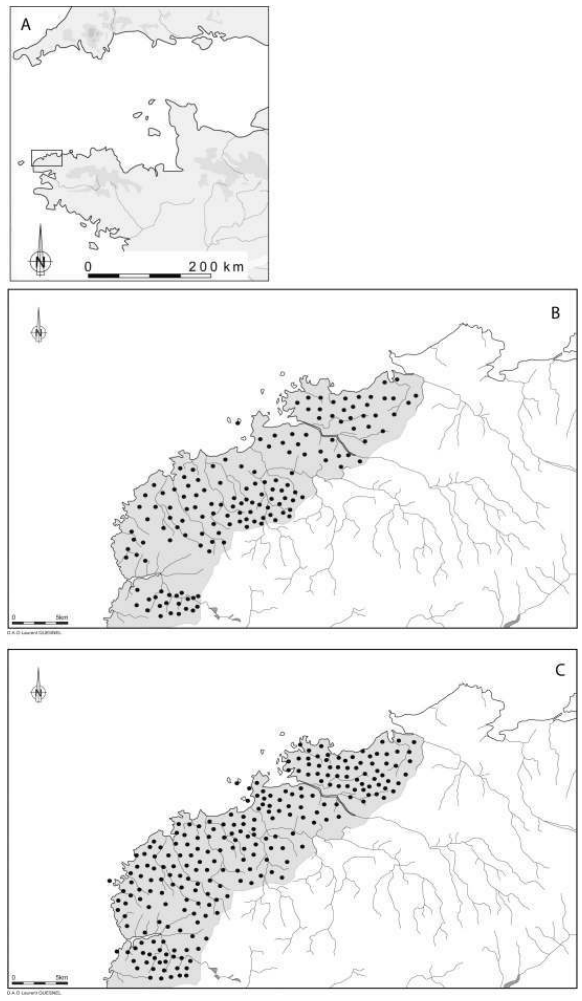

$\mathrm{Au}$ demeurant, certains estrans portent des traces d'activités qui n'auraient pu avoir lieu si les installations n'avaient été hors d'atteinte des plus hautes mers, ce qui témoigne d'un niveau marin inférieur à l'actuel d'environ $2 \mathrm{~m}$ pour le début de l'époque galloromaine (Morzadec-Kerfourn, 1995 ; Giot, 1990 ; Gaudin, 2001 ; Stéphan, 2008). Participant à l'évolution du trait de côte, d'importantes dunes vont se mettre en place, à l'Âge du Fer, et former une frange à peu près continue le long du littoral armoricain, les placages sableux pouvant atteindre des altitudes importantes sur la côte occidentale du Léon, de l'ordre de $50 \mathrm{~m}$ entre la pointe du Corsen et Lampaul-Plouarzel (Guicher et Hallégouët, 1991 ; Hallégouët, 1978, 1981 ; Morzadec-Kerfourn, 1995). 
Figure 2 : Largeur moyenne des cernes de croissance des charbons de chêne (A) et nombre de taxons trouvés dans les foyers domestiques $(B)$ des sites archéologiques du Néolithique à l'Âge du Fer en Armorique /Average width of growth rings from oak charcoals $(A)$ and number of taxa found in domestic fireplaces (B) of archaeological sites from the Neolithic to the Iron Age in Armorica

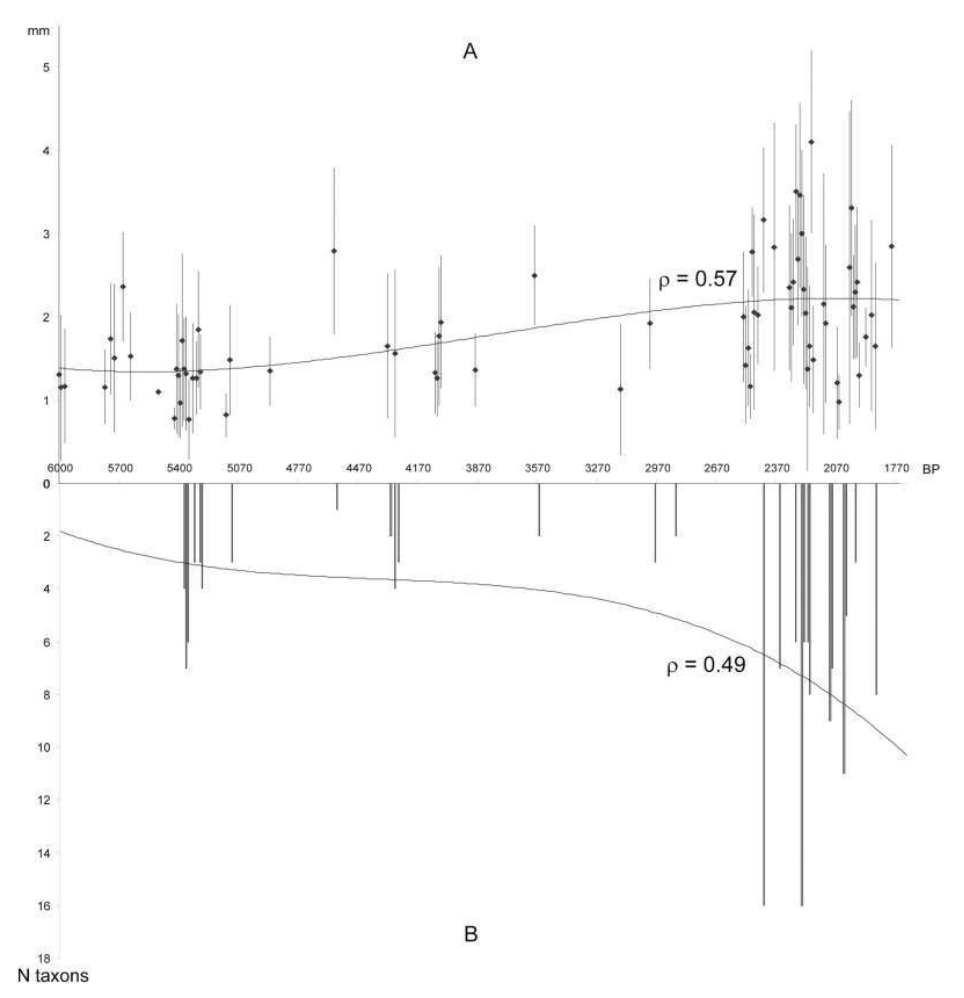

(d'après D. Marguerie et J.-Y. Hunot, 2007)

Dans la continuité de processus amorcés au Néolithique, les relations homme/milieu sont largement dominées, au cours du Ier millénaire av. n.è., par l'impact des activités anthropiques sur l'environnement, et, en premier lieu, les effets des pratiques agricoles et artisanales sur les ressources végétales, animales et minérales (Marguerie, 1990 et 1995 ; Gaudin, 2004). La confrontation des données archéologiques et paléobotaniques indique l'existence de milieux forestiers, à l'Âge du Bronze, y compris en zones littorales (Marguerie, 1992). Sur le littoral nord finistérien, M.-T. Morzadec-Kerfourn (1974) précise qu'au début de l'Âge du Bronze moyen, les défrichements sont importants; les premiers pollens de céréales apparaissent dans les spectres polliniques de tourbières. Par ailleurs, des semences carbonisées renvoient à une pratique culturale variée à base de blé amidonnier, orge polystique nue et orge polystique vêtue (Marguerie, 1992). Le site de Mez Notariou à Ouessant ${ }^{1}$ (Le Bihan et Villard, 2007) montre, pour la fin de l'Âge du Bronze et le premier Âge du Fer, un paysage insulaire déboisé à landes à genêts et ajoncs (Marguerie, 1992). Les travaux de M.-T. Morzadec-Kerfourn (1974) sur les tourbières littorales sont révélateurs d'un environnement littoral ouvert pour l'ensemble de l'Âge du Fer.

Les sites archéologiques armoricains de l'Âge du Fer, sur lesquels des données sur l'état de la végétation ont été obtenues, sont en très grande majorité des habitats. Le chêne, toujours présent en grande quantité parmi les charbons archéologiques, semble avoir été le bois de prédilection de l'exploitation forestière dans l'ouest (Marguerie, 1995). Mais si les foyers domestiques du Néolithique moyen furent presque exclusivement alimentés en 
bois de chêne caducifolié de fort calibre à croissance lente, ceux de l'Âge du Fer ont fonctionné à base d'essences beaucoup plus variées parmi lesquelles on compte bon nombre d'espèces héliophiles dont les genêts, les ajoncs, le bouleau, le noisetier et les Pomoïdées (fig. 2B). Les charbons de chênes caducifoliés, lorsqu'ils sont présents dans les foyers de l'Âge du Fer, proviennent essentiellement de branches; les troncs de chêne et les grosses branches étaient plutôt réservés à l'architecture (Marguerie, 1992, 1995).

Cependant, il semble qu'à côté de zones traditionnellement défrichées ou mises en pâtures et cultures, des forêts discontinues de type chênaies diversifiées demeuraient bien évidemment dans la région, en des lieux peu fréquentés par l'homme, ainsi que l'illustrent certaines analyses archéozoologiques qui seront développées infra (Marguerie, 1992).

\section{Évolution du peuplement en Bas-Léon et disparités spatio- temporelles}

\section{Nature, portée et limites des données archéologiques}

Les données archéologiques exploitées ici couvrent l'Holocène récent et concernent très majoritairement des sites 'terrestres'. Dans le processus archéologique tel qu'il a été longtemps conduit en France, la recherche d'une compréhension détaillée de l'évolution naturelle et temporelle de l'environnement des sites submergés est largement restée en arrière-plan de l'archéologie "terrestre ", du moins pour ce qui concerne le secteur Manche-Atlantique. Cependant, à l'instar des démarches engagées sur le pourtour méditerranéen ou encore en Centre-Ouest depuis plusieurs décennies (Burnouf et al., 1996), une évolution assez récente dans l'ouest de la France laisse augurer des perspectives intéressantes en terme de "coastal global approaches » à l'anglo-saxone. Cet aspect sera évoqué ici à travers les vestiges immergés de pêcheries d'estran qui, s'ils posent encore des problèmes de datation, peuvent être mis en perspective dans le contexte archéologique local, au moins à titre d'hypothèse.

Outre les classiques dépouillements d'inventaires archéologiques et d'archives documentaires, des approches de terrain exploitées ici sont diversifiées :

- des prospections aériennes à basse altitude ont été complétées par la photo-interprétation de missions de l'IGN, permettant principalement la détection, d'une part, de pêcheries immergées (photo 1), et d'autre part, d'enclos fossoyés, en zone agricole (Daire, 1991, 1993a, 1993b) ;

photo 1 : Double pêcherie de l'île d'Yoc'h à Landunvez (cl. P. Pondaven) 
Double fish trap near Yoc'h island, Landunvez (cl. P. Pondaven)

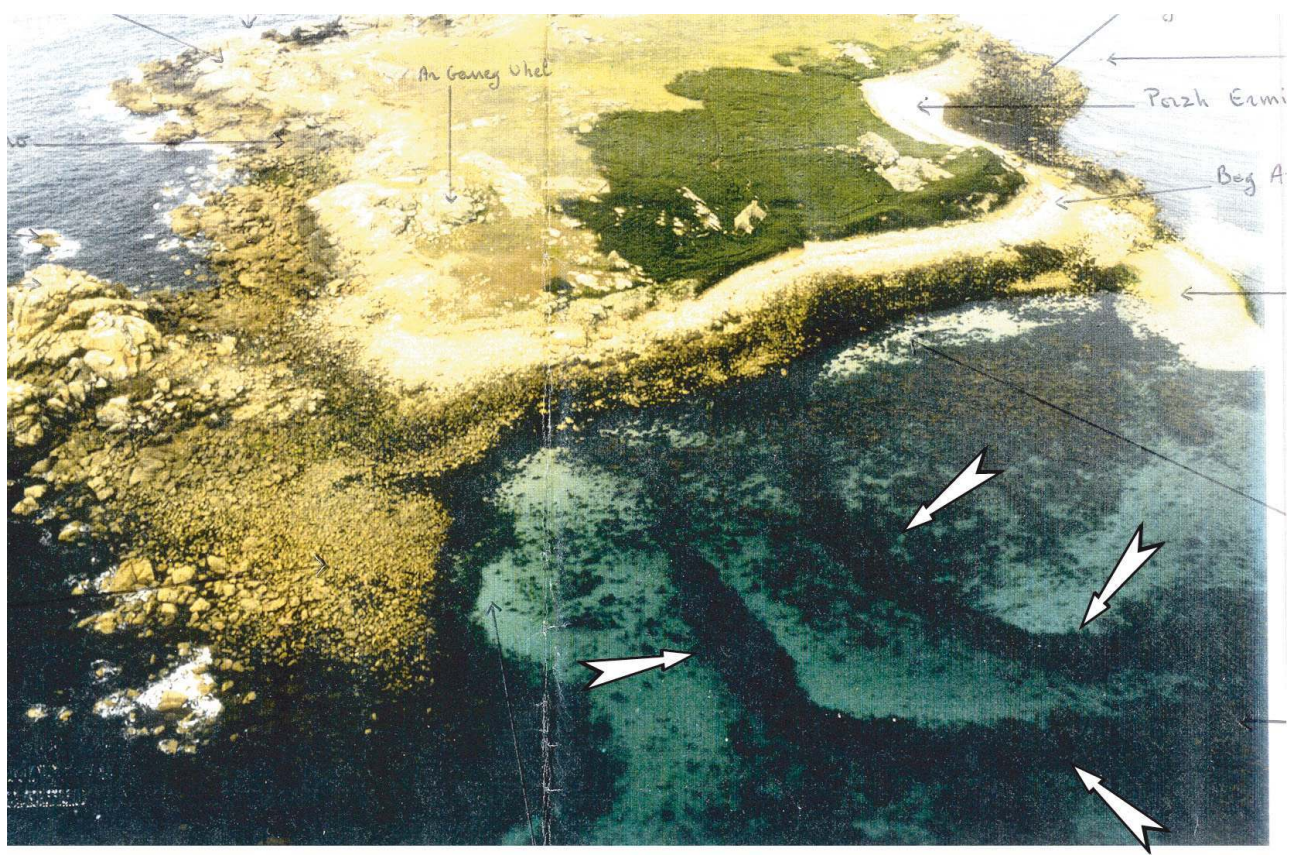

- certains sites d'enclos découverts par voie aérienne ont fait l'objet de vérifications au sol, après labours, de manière à en préciser la nature et la chronologie, ce qu'illustrent ici les structures complexes de Kernizan à Plouguin, dont les prospections au sol ont confirmé l'attribution chronoculturelle (fin de la Protohistoire et époque gallo-romaine) (photos 2 et 3) ;

Photo 2 : L'enclos de Kernizan à Plouguin, vu d'avion (cl. M.-Y. Daire)

The Kernizan enclosure (Plouguin), view from the air (cl. M.-Y. Daire)

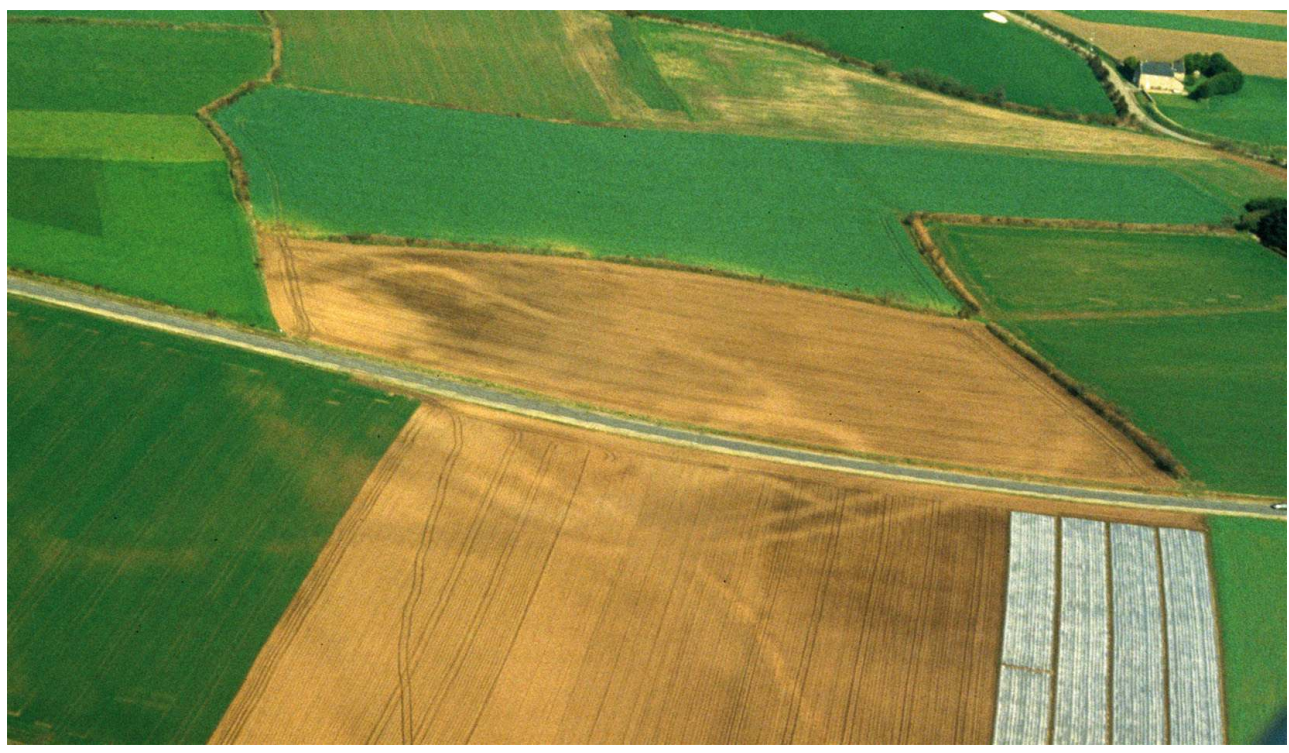

Photo 3 : L'enclos de Kernizan à Plouguin, vue d'un des fossés (cl. M.-Y. Daire) 
The Kernizan enclosure (Plouguin), view with feet on the ground (cl. M.-Y. Daire)

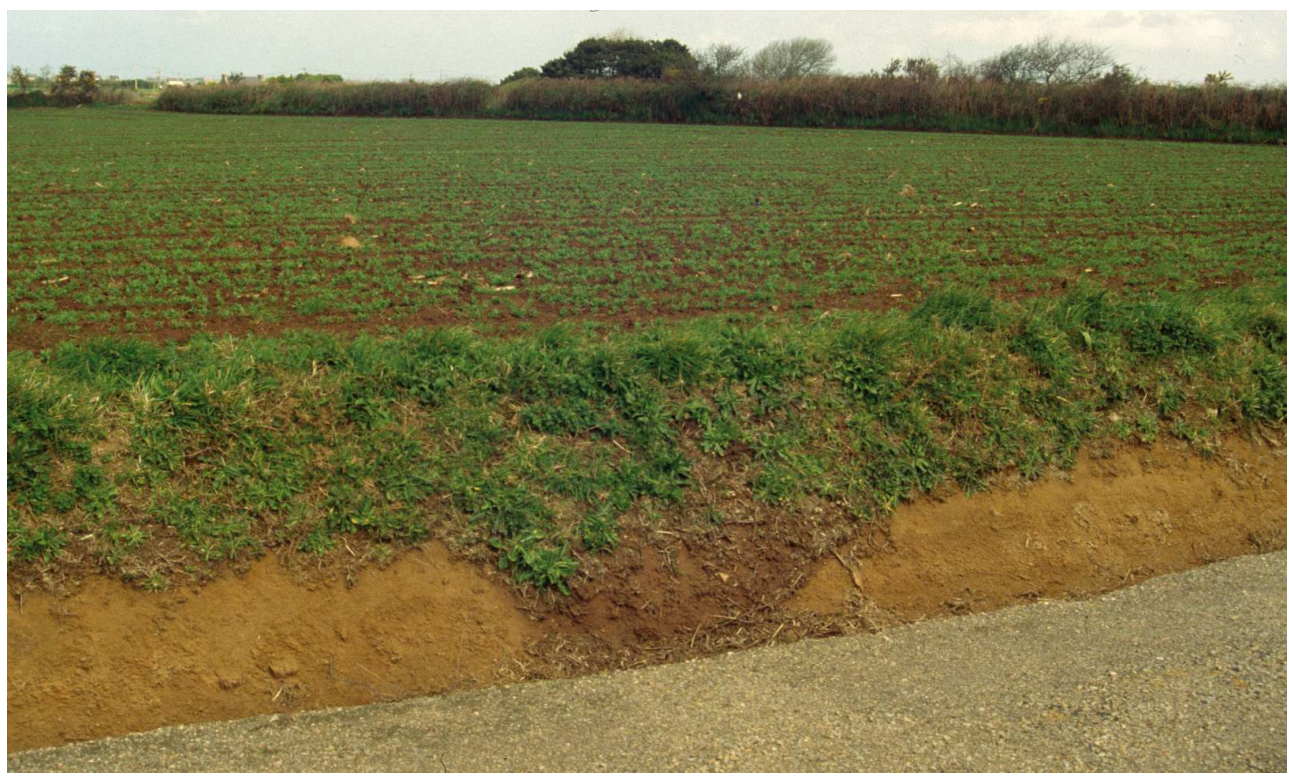
peu plus de 350 sites ou gisements archéologiques pris en compte ici sont susceptibles d'illustrer les modalités de peuplement de cette région au cours du Ier millénaire av. n.è.: dont 231 datent de l'Âge du Fer, 15 seulement de l'Âge du Bronze final et 9 du début de l'époque gallo-romaine (tableau 1). Cet échantillonnage montre une hétérogénéité des données selon les périodes avec, par exemple, des structures d'habitat dont on ne sait pratiquement rien avant le second Âge du Fer. Autre limite évidente dans la qualité des informations disponibles, dans le domaine funéraire cette fois, avec des centaines de stèles funéraires datant de la période ve-IIIe siècles av. n.è., révélatrices de la densité d'une population dont les cimetières nous sont très mal connus, ces stèles étant généralement déconnectées de leurs contextes archéologique d'origine. Ce corpus souffre aussi d'inégalités dans la répartition géographique de l'information; les massifs dunaires évoqués supra agissent à la fois comme protection mais aussi comme "masque » de vestiges archéologiques qui restent à découvrir.

24 Les données concernant l'Âge du Bronze sont mal calées chronologiquement par comparaison avec celles de l'Âge du Fer. Les sites attribués au Bronze final en particulier sont assez peu nombreux par rapport à la totalité des structures datées de l'Âge du Bronze. En effet, si cette période est envisagée dans sa totalité, le nombre de sites passe 
alors à 149 (tableau 1, fig. 1-B) : 116 relèvent du monde funéraire, 26 sont des dépôts ou des objets isolés métalliques, et seulement 7 traduisent des occupations domestiques.

Les sites funéraires, principalement des tombes sous tumulus, sont très nombreux dans ce secteur comme le démontre leur très forte concentration dans la commune de Plouguin (Fily, 2008). Il apparaît difficile d'établir des correspondances dans le temps entre les sites et de définir exactement lesquels sont contemporains. Seuls les tumulus du Bronze ancien, attribués au groupe de la première série telle que définie en 1951 par P.-R. Giot et J. Cogné, sont bien identifiés grâce à leur mobilier caractéristique : poignards en bronze à petits clous d'or, et pointes de flèches en silex notamment. Quelques tombes sont attribuées au Bronze moyen, mais aucune n'est datée avec certitude du Bronze final. Beaucoup de coffres recouverts d'un tertre sont simplement attribués à l'âge du Bronze sans plus de précisions chronologiques.

Tableau 1 : Répartition géographique et chronologique des données utilisées dans l'étude

Geographical and chronological distribution of the data used in the study

\begin{tabular}{|c|c|c|c|c|c|c|}
\hline Commune & $\begin{array}{ll}\text { Âge } & d u \\
\text { Bronze } & \end{array}$ & $\begin{array}{l}\text { dont attestés } \\
\text { de l'Âge du } \\
\text { Bronze final }\end{array}$ & $\begin{array}{l}\text { Âge du } \\
\text { Fer }\end{array}$ & $\begin{array}{l}\text { Antiquité } \\
\text { précoce }\end{array}$ & $\begin{array}{l}\text { Non } \\
\text { daté }\end{array}$ & Total \\
\hline Brélès & 0 & 0 & 9 & & 5 & 14 \\
\hline Guissény & 9 & 1 & 11 & & 1 & 21 \\
\hline Lampaul-Plouarzel & 1 & 1 & 3 & & 2 & 6 \\
\hline $\begin{array}{l}\text { Lampaul- } \\
\text { Ploudalmézau }\end{array}$ & 1 & 1 & 7 & 1 & 3 & 12 \\
\hline Landéda & 5 & 1 & 20 & & 8 & 33 \\
\hline Landunvez & 6 & 0 & 17 & & 12 & 35 \\
\hline Lanildut & 1 & 0 & 1 & & 2 & 4 \\
\hline Lannilis & 12 & 2 & 10 & & 6 & 28 \\
\hline Plouarzel & 19 & 1 & 26 & & 13 & 58 \\
\hline Ploudalmézeau & 18 & 3 & 20 & & 6 & 44 \\
\hline Plouguerneau & 20 & 3 & 51 & 4 & 12 & 87 \\
\hline Plouguin & 34 & 1 & 16 & 3 & 11 & 64 \\
\hline Plourin & 12 & 0 & 18 & & 6 & 36 \\
\hline Porspoder & 6 & 0 & 11 & 1 & 10 & 28 \\
\hline St Pabu & 4 & 1 & 9 & & 1 & 14 \\
\hline Tréglonou & 1 & 0 & 2 & & 1 & 4 \\
\hline Total & 149 & 15 & 231 & 9 & 99 & 488 \\
\hline
\end{tabular}

Contrairement aux indices d'habitats et aux édifices funéraires, les dépôts d'objets métalliques sont bien calés chronologiquement par la typologie de leur mobilier (Giot et 
al., 1995). Ce phénomène apparaît à partir du Bronze moyen et se multiplie au Bronze final. Il se poursuit au premier Âge du Fer par l'enfouissement de dépôts de haches à douille dites «armoricaines ", dont la caractéristique principale est leur forte teneur en plomb qui empêche tout usage pratique en tant qu'outil. Plusieurs dépôts de l'horizon de Tréboul ainsi que des dépôts de haches à talon de la phase récente du Bronze moyen sont connus dans le secteur considéré par l'étude. Pour les phases anciennes et moyennes du Bronze final, aucun dépôt de l'horizon de Rosnoën, ou de Saint-Brieuc-des-Iffs n'a été découvert. Une dizaine de dépôts ou objets isolés métalliques de l'horizon de l'épée en langue de carpe datés de la fin du Bronze final sont par contre inventoriés dans le plateau littoral du Bas-Léon. Ces dépôts de quantité variée (de quelques objets à plus d'une centaine) contiennent la plupart du temps les éléments classiques de ce groupe métallique caractéristique du Bronze final Atlantique : des armes, des outils, des éléments de toilette et de parure, des pièces de harnachement et de char, et enfin des éléments de métallurgie comme des déchets de fonderie et des lingots. Deux dépôts de hache à douille de type armoricain sont également répertoriés dans le plateau littoral du Bas-Léon. Si les dépôts sont bien calés dans le temps, leur localisation précise dans le paysage est souvent méconnue. Ils proviennent souvent de découvertes anciennes, du xixe ou du début du XX e siècle, et les découvreurs ne notaient alors que très rarement les coordonnées géographiques ou les numéros des parcelles des découvertes. Seul l'objet avait de la valeur à leurs yeux, et non sa connexion avec un lieu. Il est toutefois possible de proposer des indices de localisation pour une large partie d'entre eux, notamment d'après les lieuxdits des découvertes, mais il convient de rester prudent. Resituer ces dépôts dans le paysage apparait pourtant fondamental dans la recherche actuelle, et devrait permettre de mieux comprendre ce phénomène dont la fonction divise encore la communauté des archéologues, même si l'hypothèse de dépôt rituel est largement privilégiée aujourd'hui.

Yoc'h et Guennoc : deux territoires 'insulaires' emblématiques 
Figure 3 : Carte de localisation de l'île d'Yoc'h et de l'île Guennoc et des principaux sites insulaires mentionnés dans le texte (DAO L. Quesnel)/Location of Yoc'h and Guennoc islands and the main island sites mentioned in the text (DAO L. Quesnel)

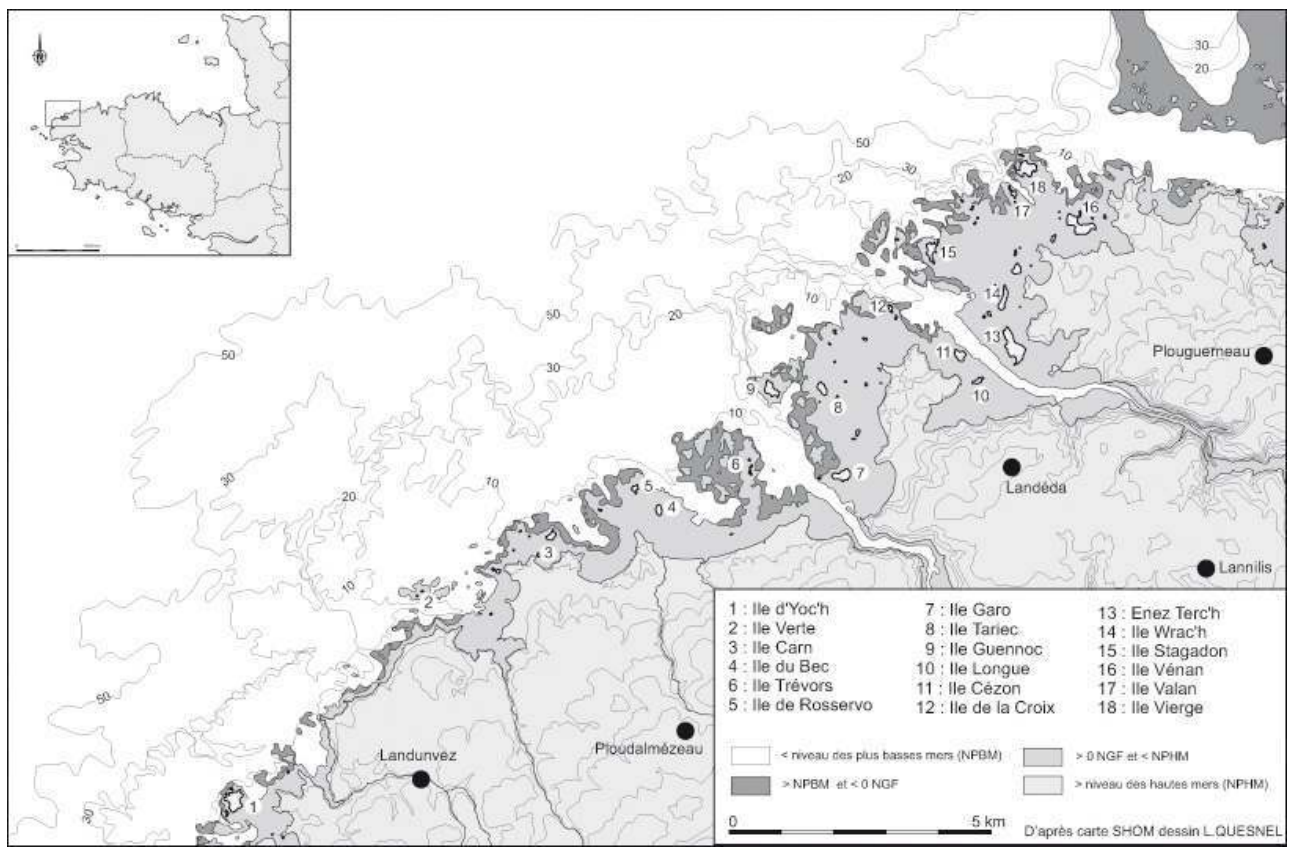

Territoires aujourd'hui insulaires, Yoc'h et Guennoc présentent beaucoup de points communs, tant dans les rythmes et formes de leurs peuplements successifs, que dans l'état de conservation de vestiges protégés du fait d'un isolement progressif de ces îles, où les relations anciennes entre l'homme et le milieu sont ainsi plus palpables et cohérentes (fig. 3).

L'̂̂le d'Yoc'h à Landunvez a connu une importante occupation humaine de la fin de l'Âge du Fer, du début du ier siècle av. n.è. au milieu du ier siècle de n.è. sur l'ensemble de son territoire aujourd'hui réduit à un peu plus de 7 ha (Daire, 2001) (photos 4 et 5). Les fouilles y ont révélé l'existence d'un village dont cinq bâtiments en pierre sèche ont été étudiés dans leur environnement immédiat. Les activités alors pratiquées sur l'île étaient diversifiées : la production de sel, à partir de l'eau de mer, était mise en œuvre au sein d'un atelier caractéristique de cette période (Daire, 2003), en complément des activités agricoles ou d'autres artisanats (travail du lignite, pêche, etc.). Les constructions gauloises sont directement implantées sur un complexe funéraire mégalithique (cairn néolithique et dolmen à couloir) qui a manifestement servi de carrière de pierres aux occupants de l'Âge du Fer. 
Photo 4 : Talus de l'Âge du Fer sur l'île d'Yoc'h (cl. M.-Y. Daire)/Iron Age rampart on Yoc'h island (cl. M.-Y. Daire)

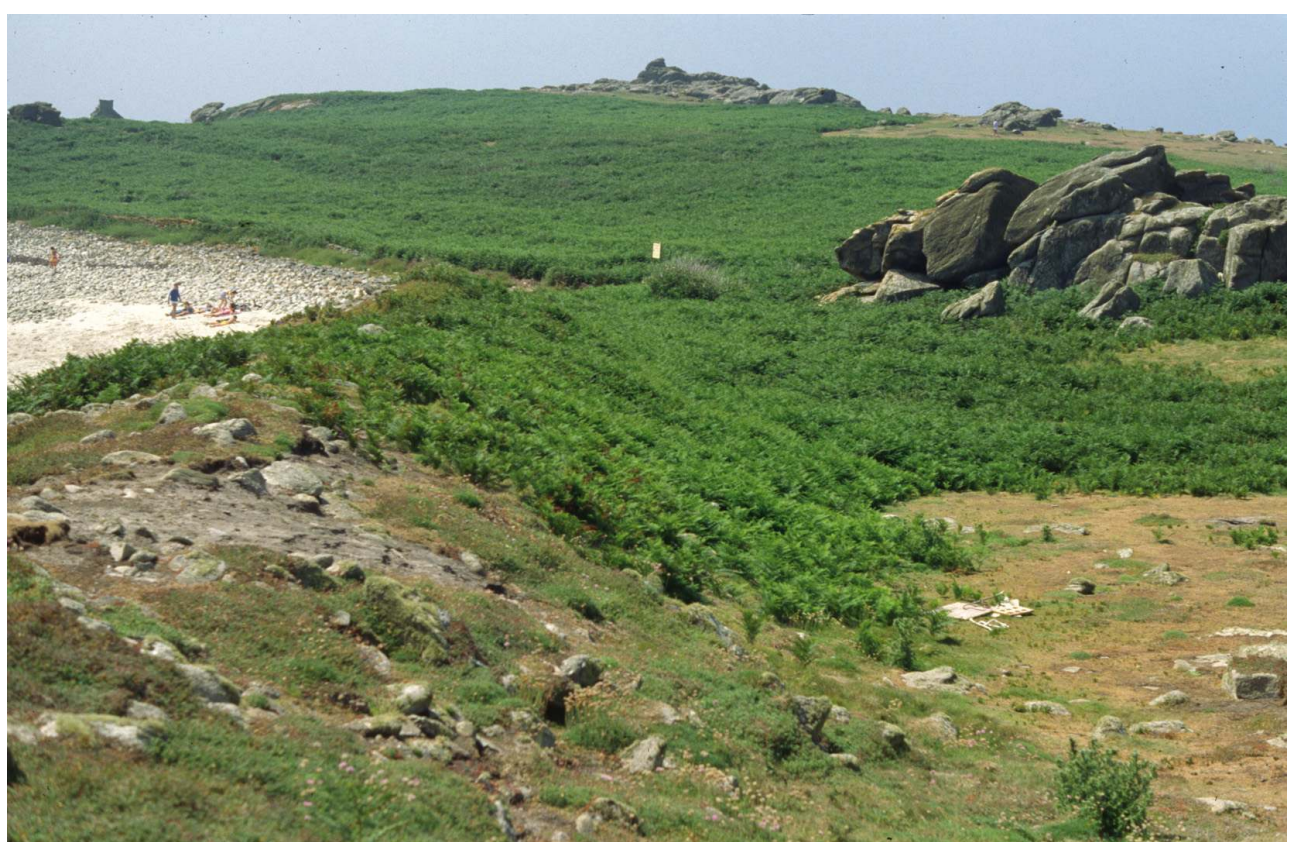

Photo 5 : Le hameau de la fin de l'Âge du Fer de l'île d'Yoc'h en fin de fouille (cl. M.-Y. Daire)/The Iron Age hamlet on Yoc'h island, view at the end of the excavation (cl. M.-Y. Daire)

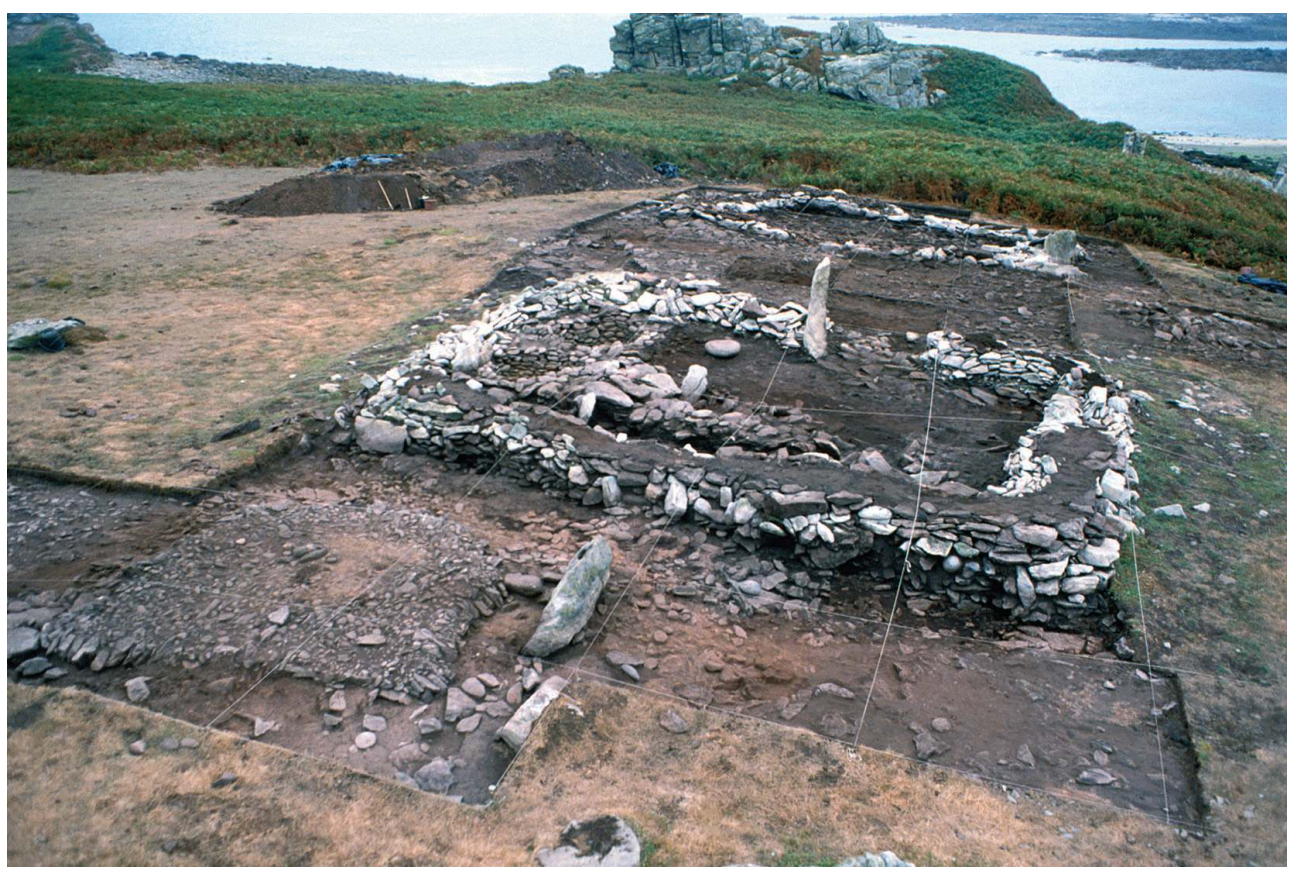

L'analyse anthracologique des charbons de bois provenant de diverses structures a révélé la présence et l'exploitation d'espèces typiques de la lande armoricaine (ajoncs, genêt et noisetier) ayant servi de combustibles dans les foyers domestiques et artisanaux du site, tandis que les restes de chêne et de frêne correspondent à des grosses branches ou des troncs issus d'éléments d'architecture (charpente ou poteaux porteurs) (Marguerie, 1992). En revanche, les vestiges organiques non brûlés sont largement absents du niveau archéologique qui affleure sous la pelouse littorale, peu propice à leur conservation ; en 
effet, les analyses sédimentologiques ont souligné que l'acidification des sols autour du site est un phénomène lié à l'anthropisation de l'environnement, antérieur probablement à l'Âge du Fer (Gebhardt, 1989).

30 Au cours des fouilles menées sur l'île Guennoc à Landéda dans les années 1960, P.-R. Giot s'intéressa principalement à la série des 4 cairns mégalithiques, mais reconnut plusieurs autres phases d'occupation humaine (Giot, 1987). Un dépôt de l'Âge du Bronze final de l'horizon métallique de l'épée en langue de carpe y mentionné pour la première fois par P. Du Chatellier (1891 et 1899), est connu sur l'île Guennoc. "Fortifié » sur son flanc oriental par un talus rempart (photo 6), ce territoire de plus de 5 ha a accueilli, à la fin de l'Âge du Fer (fin du IIe siècle av. n.è.-début du ier siècle de n.è.) un groupe humain qui a édifié au moins 8 bâtiments, pour partie ceinturés par un petit talus d'enclos (Daire et Quesnel, 2008). Comme à l'île d'Yoc'h, ces constructions en pierre sèche bénéficièrent de la récupération des matériaux des monuments mégalithiques antérieurs; sous la forme d'un souterrain, une structure de stockage complétait les installations domestiques.

Photo 6 : Coupe dans le talus de l'île Guennoc (noter la présence d'un fragment de meule rotative) (cl. P.-R. Giot, archives du labo. Archéosciences, Université de Rennes 1)/Excavation trench in the rampart of Guennoc island (notethe presence of a broken millstone) (cl. P.-R. Giot, document Archéosciences lab, Rennes 1 University)

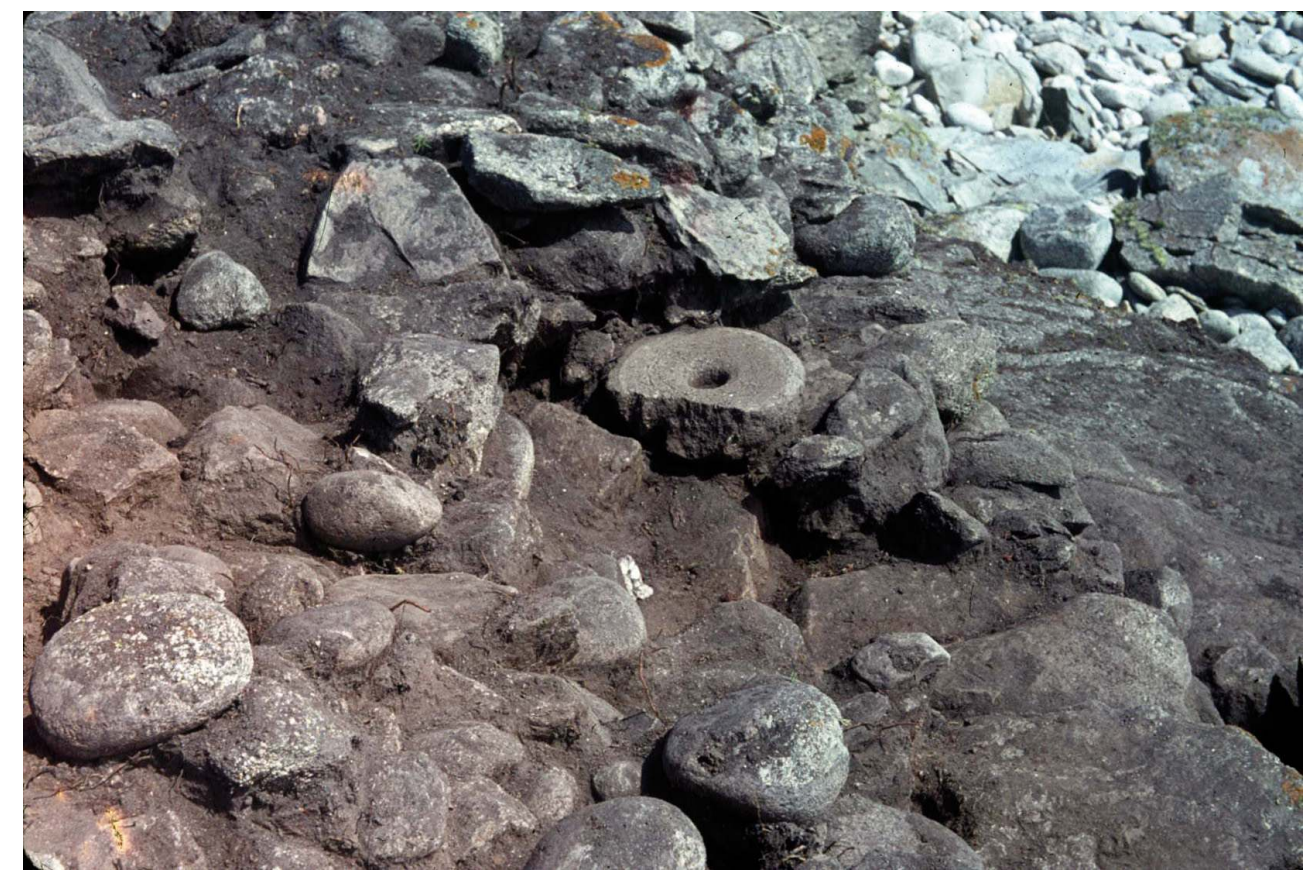

Sur le plan quantitatif, d'après le nombre de bâtiments reconnus et l'importance du mobilier recueilli, on peut imaginer que dans les deux cas ce sont quelques «familles » (une trentaine de personnes au maximum de manière synchrone) qui ont pu vivre sur ces territoires plus étendus qu'aujourd'hui, en pratiquant des activités diversifiées.

L'élevage de caprinés est probable sur ces îles, comme c'est souvent le cas sur les côtes de l'ouest de la Gaule (Baudry, 2005). Il semble compléter les activités de cueillette et de pêche des invertébrés et vertébrés marins (coquillages, crustacés, poissons, etc.) ; de plus la découverte de nombreuses fusaïoles témoigne d'une activité de filage de fibres qui peuvent aussi bien être d'origine animale que végétale. La présence de chevaux est, quant à elle, attestée sur l'île d'Yoc'h par la découverte d'un mors de cheval qui n'exclut pas que les équidés aient été successivement utilisés pour le travail et leur force motrice, en 
relation avec les travaux agricoles puis pour la consommation alimentaire. La présence d'un squelette de vache, dans le couloir d'un des dolmens de l'île Guennoc, peut également être mentionnée, laissant envisager la possibilité d'un élevage de bovinés sur ce site (Daire, 2008).

$\mathrm{Au}$ titre des autres pratiques agricoles, nous évoquerons les trois (demi) meules rotatives trouvées dans le corps du talus-rempart (photo 6) ainsi que dans les maisons de l'enclos attestant d'une activité de broyage, de céréales ou d'autres matières, végétales ou non, mais aussi les remarquables traces "fossiles" de cultures en billons, tout à fait comparables sur les deux îles et qui, sur l'île d'Yoc'h, ne peuvent que remonter à l'Âge du Fer (cf. infra).

\section{Évolution spatio-temporelle du peuplement}

Les données disponibles relèvent principalement des modalités d'occupation (habitat et activités agricoles ou artisanales) diversement illustrées selon les périodes, mais aussi des réseaux de contacts contemporains.

Les habitats reconnus ici datent majoritairement de l'Âge du Fer et du début de l'Antiquité. La faible représentativité des habitats du Bronze moyen et final sur le littoral armoricain est imputable, selon P.-R. Giot, à la recherche de gisements d'étain, de plomb et au besoin en combustible générés par le développement de la métallurgie et qui auraient davantage attiré les populations vers l'intérieur des terres (Giot et al., 1995). Cette affirmation doit être fortement nuancée, car les tombes sous tumulus, et les nombreux dépôts, témoignent d'une implantation ancienne et importante dans ce secteur pendant l'Âge du Bronze. Si les habitats sont aussi peu nombreux, il semble que l'une des raisons soit d'ordre méthodologique, en particulier liée à l'absence de grands décapages extensifs développés dans le cadre de l'archéologie préventive. Les habitats de cette période découverts récemment dans le centre de la Bretagne par l'INRAP en sont le meilleur témoin.

Un autre élément d'explication pour ce déficit en habitats côtiers de l'Âge du Bronze et du premier Âge du Fer est peut-être lié à l'épisode de transgression marine qui se poursuit encore aujourd'hui, des habitats installés sur des portions basses de la côte ayant pu se trouver submergés par la suite (Stéphan, ce volume); au second Âge du Fer, les communautés littorales ont souvent recherché des points relativement hauts de la frange côtière, bénéficiant d'un large panorama et en particulier d'un point de vue sur le paysage maritime ; les habitats de l'île d'Yoc'h et de Guennoc répondent particulièrement bien à ces critères, ce qui souligne l'importance des possibilités de contacts visuels dans certaines implantations.

On peut s'interroger sur une éventuelle attribution chronologique à l'Âge du Bronze, sur des critères morphologiques, de la petite série d'enclos circulaires identifiés à Kerrincuff (Lampaul-Ploudalmézeau), Toul al Louarn (Lannilis), Kergros et Lanrivanan (Plouguin), dont les superficies réduites varient de 0,05 ha à 0,9 ha.

Les occupations de l'Âge du Fer, dans ce secteur géographique, prennent des formes diversifiées qui se traduisent dans les formes de l'habitat: hameaux ou villages côtiers (Yoc'h, Guennoc, île du Bec, Trévorc'h, etc.) (Galliou, 2010), avec parfois un caractère défensif très marqué (éperon barré de Kermorvan par exemple), enclos fossoyés souvent associés à des souterrains (une quinzaine dans la zone considérée). 
39 La conquête romaine ne semble pas avoir eu de retombées immédiates sur les habitats côtiers de ce secteur géographique, relativement "périphériques " par rapport à des centres urbains tels que Kérilien à Plouneventer, dans la mesure où les traditions architecturales et artisanales semblent s'être poursuivies jusqu'à une date avancée dans le $\mathrm{I}^{\mathrm{er}}$ siècle de n.è.

40 Dans le domaine funéraire, de nombreuses stèles (160 dans le secteur considéré) ont appartenu à des nécropoles comme celle de Pembrat-Vihan à Lannilis, majoritairement datées des ve-Ive siècles av. n.è. et leur densité semble révélatrice d'un peuplement régional assez dense à la fin du premier Âge du Fer (Daire, 2005).

41 Les côtes, en général, et celles du nord du Finistère tout particulièrement, ont joué au cours de ce $\mathrm{I}^{\mathrm{er}}$ millénaire av. n.è. un rôle fondamental dans les échanges à plus ou moins longues distances, en tant qu'interface «terre-mer» ou aboutissent (et commencent) routes maritimes et routes terrestres. Ceci se traduit à la fin de l'Âge du Bronze par le fait que les dépôts « atlantiques » regroupent des productions locales et des importations. Les dépôts de la fin du Bronze final contiennent ainsi de nombreux objets similaires tout le long de la façade atlantique : les fragments de lame du type de l'épée en langue de carpe en sont les principaux exemples. Ils témoignent notamment de la réalité de l'axe Atlantique, de la péninsule ibérique à l'Écosse, et des échanges culturels et commerciaux entre ces provinces reliées par la mer. Des éléments exogènes viennent confirmer des échanges plus lointains, notamment avec les pays nordiques, comme en témoigne la hache de type nordique du dépôt de l'île Guennoc (Briard et Onnée, 1996).

Ce rôle de vecteur ne se démentira pas au cours de l'Âge du Fer: la découverte, sur la plage de Lampaul-Ploudalmézeau, d'un statère en or de Cyrène (ville de l'actuelle Lybie) frappé entre -322 et -315 apparaît dès lors comme une confirmation matérielle des voyages de Pythéas le Grec (vers 320-300 av. n.è.) (Giot et al., 1995). La présence systématique d'amphores originaires d'Italie témoigne d'échanges à longue distance à la fin du IIe siècle av. n.è., qui devaient au moins pour une part d'entre eux emprunter des voies maritimes de circulation. Mais les circulations par voie terrestre sont également illustrées dans ce secteur géographique, par un réseau dense de voies antiques et de chemins (Pape, 1978; Galliou, 2010) qui apparaît comme un élément puissant dans la structuration du paysage (cf. infra) (Le Pennec, 2007).

\section{Dynamiques du peuplement et du paysage}

\section{Quand l'environnement conditionne le peuplement...}

\section{Appel de la côte et appel du large...}

Les dynamiques paysagères peuvent être difficiles à cerner sur un pas de temps relativement court (pour l'archéologue) qui est celui d'un millénaire ; certaines données relatives aux évolutions du trait de côte, aux contextes sédimentaires, ou encore à la nature et la biodiversité animale et végétale de la bande côtière peuvent y contribuer.

La régression marine apparente du $\mathrm{I}^{\mathrm{er}}$ millénaire av. n.è., a libéré de larges estrans sableux, peu ou pas exploités (à l'évidence) au cours des siècles précédents et que l'homme va pouvoir mettre à profit (Giot, 1990). Les ressources biologiques y sont variées et la cueillette est assez simple à mettre en œuvre et efficace dans une région où le marnage est de $9 \mathrm{~m}$ à l'heure actuelle: collectes et consommation de coquillages, de 
crustacés, pêche côtière, qui sont attestées à l'île du Bec à Lampaul-Ploudalmézeau et à l'île Trévorc'h à Saint-Pabu (AMARAI, inédit) ou encore à l'île Guennoc où les $10 \mathrm{~m}^{3} \mathrm{de}$ coquilles accumulées à la fin de l'Âge du Fer dans l'un des dolmens témoignent d'une occupation prolongée des lieux par un groupe humain relativement fourni (Giot, 1987; Daire et Quesnel, 2008).

Ces espaces intertidaux furent aussi mis à profit pour l'implantation de pêcheries ou barrages à poissons, une trentaine dans le pays des abers, dont une partie date très vraisemblablement de la période qui nous intéresse ici si l'on en croit certains contextes archéologiques et critères d'installation (Daire, 2008). C'est le cas par exemple pour la double pêcherie de l'île d'Yoc'h qui, compte tenu de sa cote d'implantation (-3,60 m NGF soit $+0,30 \mathrm{~m} /$ zéro SHOM) et dans la perspective d'une élévation du niveau marin d'environ $2 \mathrm{~m}$ depuis le début de notre ère (Giot, 1990), a probablement été construite et utilisée par la communauté gauloise implantée sur le site au $\mathrm{I}^{\mathrm{er}}$ siècle av. n.è. et au Ier siècle de n.è. La pêcherie identifiée à proximité de l'île Venan (Plouguerneau) est peutêtre à mettre en relation avec les tombelles (de l'Âge du Bronze ou de l'Âge du Fer) reconnues sur l'îlot de même qu'à l'île Segal (Plouarzel), coexistent un barrage à poissons en pierre et un gisement domestique du second Âge du Fer. Cette exploitation des ressources halieutiques se poursuivra localement à l'époque gallo-romaine comme en attestent les viviers à poissons gallo-romains de Guissény (Galliou, 2010).

Par ailleurs, l'accès à la ressource en eau de mer salée a, pour partie, conditionné l'implantation des ateliers de bouilleurs de sel de l'île d'Yoc'h à Landunvez, de Beg ar Vil à Lampaul Plouarzel et de l'île du Bec à Lampaul-Ploudalmézeau, en même temps que la disponibilité des autres matières premières indispensables que sont l'argile, d'une part, et les combustibles, d'autre part ; mais, sur ce dernier point, nous avons vu que la végétation de lande et les algues fournissent des alternatives intéressantes confirmées par les expérimentations (Daire et al., à paraître).

Enfin, il est évident que la portion basse de la côte léonarde, avec ses multiples indentations formant autant d'abris potentiels, a fourni des possibilités d'échouage pour les navires antiques et pré-romains et que, dès lors, elle peut être considérée comme une zone de contacts privilégiés, ce dont témoignent divers vestiges archéologiques évoqués supra. Ce potentiel a dû fortement contribuer à attirer des implantations humaines à des périodes où l'économie est tournée vers les échanges et les contacts.

\section{Analyses de la dynamique autour de l'aber Ildut}

Nous avons souligné le fait que les abers font partie des éléments les plus marquants du paysage du Bas-Léon. L'importance stratégique de l'aber Ildut est peut-être à considérer en relation avec l'exploitation des gisements d'étain alluvionnaire de Saint-Renan, attestée à l'époque gallo-romaine mais qui remonte probablement à la Protohistoire, cet axe fluvio-maritime constituant un débouché possible vers un trafic par voie maritime.

À partir des découvertes aériennes de fossés d'enclos, particulièrement denses dans ce secteur (Daire, 1991, 1993a), une expérience de lecture du paysage ancien a été menée autour de l'aber Ildut sur une surface d'environ $40 \mathrm{~km}^{2}$ où sont recensées une quinzaine de structures (Arbousse-Bastide, 2000). Par rapport à une topographie dont l'altitude maximale est localement de $75 \mathrm{~m} \mathrm{NGF}$, les enclos sont implantés à des paliers dont les altitudes varient de 25 à $35 \mathrm{~m}$ et de 65 à $70 \mathrm{~m}$ NGF, tandis que la moyenne des sites se trouve entre 40 et $60 \mathrm{~m}$ NGF. Il s'agit d'implantations de plateaux ou de petits 
promontoires en surplomb de vallée, souvent à proximité de cours d'eau; dans l'ensemble, les sites sont exposés dans un cadran sud/sud-est et rares sont les exemples situés sur des pentes orientées à l'ouest.

Deux systèmes principaux d'organisation du paysage antique semblent émerger de l'interprétation spatiale des données (fig. 4).

Figure 4 : Cartographie des enclos 'protohistoriques' autour de l'aber Ildut (d'après ArbousseBastide, inédit, DAO L. Quesnel)/Mapping of the 'protohistoric' type enclosures around the Aber Ildut (after, Arbousse-Bastide, unpublished, DAO L. Quesnel)

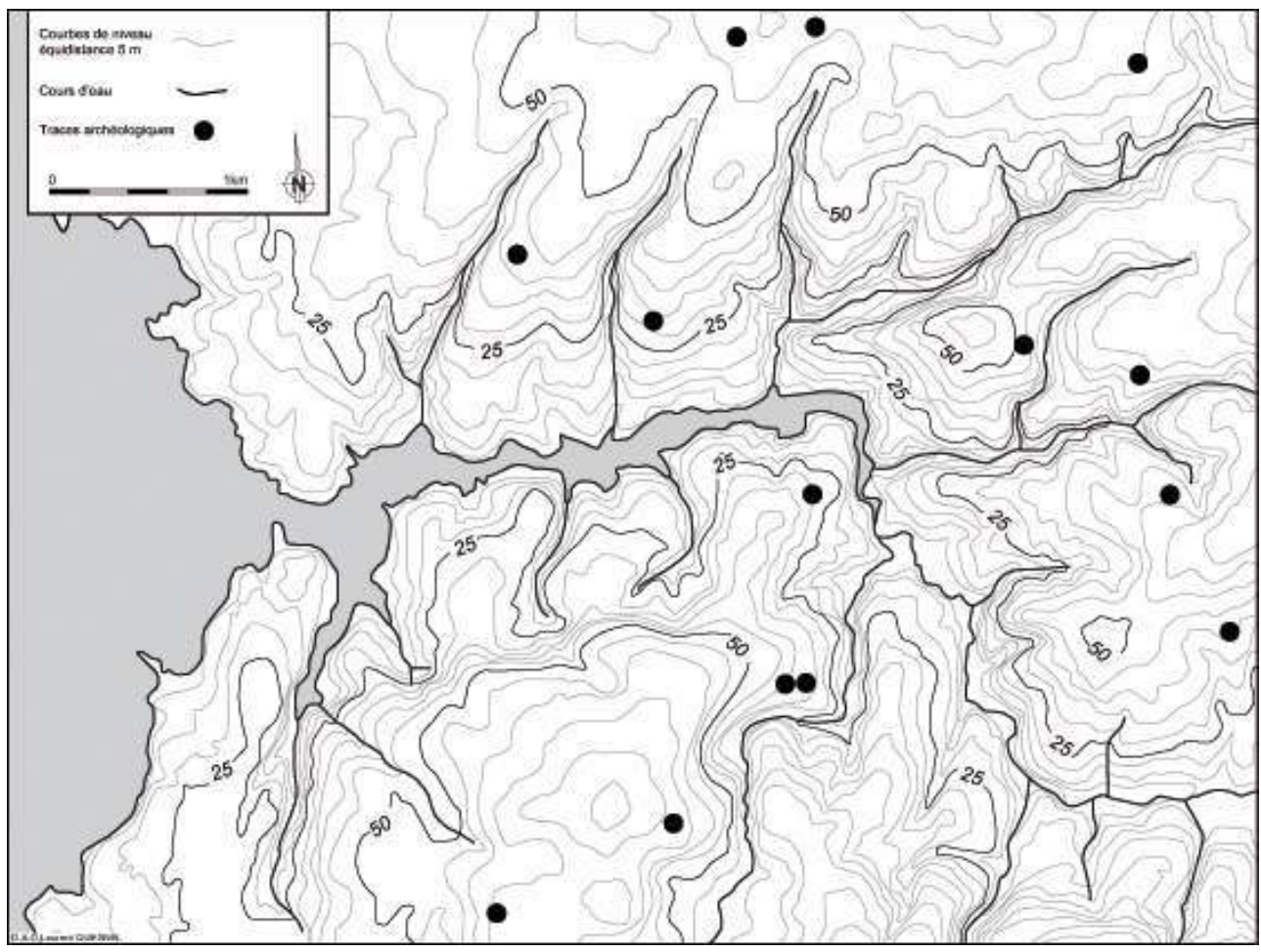

Dans la partie nord, une série de vallées de cours d'eau forment des dénivelés importants divisant naturellement le paysage en une série de bandes dont le module moyen est de $1 \mathrm{x}$ $2 \mathrm{~km}$ orientés N/S perpendiculairement au bras principal de l'Aber dans la partie ouest et plus inclinés dans la partie orientale. L'implantation des enclos, dans cette zone, semble avoir mis à profit l'organisation naturelle de ce paysage puisque, à chaque bande de terre correspond un enclos situé soit au centre à mi-pente (entre 30 et $40 \mathrm{~m}$ d'altitude), soit au sommet entre les sources des cours d'eau.

Dans la partie au sud de l'Aber, les enclos s'organisent de manière beaucoup plus hiérarchisée, évoquant une organisation spatiale réfléchie, basée sur une distribution régulière à partir d'un centre symbolisé par un enclos principal de taille plus importante et au plan complexe. L'enclos de Kervéatous à Lampaul-Plouarzel (fig. 5), au plan paracurviligne à fossés multiples, domine par sa taille (2,5 ha) les structures du secteur, bien que sa situation ne soit pas culminante en terme de topographie (entre 25 et $35 \mathrm{~m}$ d'altitude NGF) ; de plus, son implantation paraît stratégique, à l'interface de la campagne et d'une voie de communication maritime prolongée par l'Aber. Au sud de cet enclos, suivant la vallée assez encaissée d'un cours d'eau, une série de trois autres enclos forme une chaîne orientée NO/SE aux maillons implantés à égales distances les uns des autres $(1 \mathrm{~km})$ et occupant de petits promontoires rocheux. 


\section{Changements globaux, flux migratoires et « toutes ces sortes de choses »...}

53 L'île d'Yoc'h est aujourd'hui séparée du continent par un bras de mer d'un peu plus de $500 \mathrm{~m}$, ce qui en limite l'accès à pied sec aux seules marées basses de vives eaux, soit quelques jours par an. Un tombolo reliant l'île à la pointe d'Argenton est encore dénommé « Le Pont de l'île »; en effet, il s'agit bien d'un territoire récemment insularisé, mais qui, à la fin de l'Âge du Fer, n'était encore qu'une presqu'île. Une telle situation topographique expliquerait la présence d'un fort talus-rempart conservé encore aujourd'hui sur une hauteur de 5 à $6 \mathrm{~m}$ et qui barre toute la face continentale de l'île ; une même observation a été formulée pour Guennoc qui possède aussi son talus rempart tourné en direction du continent (Daire, 2008). À Yoc'h comme à Guennoc, il s'agit davantage de structurer l'espace, de contrôler les accès (des animaux en particulier) que d'établir une réelle " défense » (Daire, 2001 ; Daire et Quesnel, 2008). Alors qu'elles étaient densément occupées à partir de la fin du IIe siècle av. n.è., ces deux îles ont été abandonnées dans le courant du Ier siècle de n.è.; la cause de cette désertion est peutêtre à chercher dans les conséquences de changements climatiques et environnementaux (Stéphan, ce volume) : une montée relative du niveau marin, évaluée à $2 \mathrm{~m}$ entre le changement d'ère et l'actuel (Giot, 1990), combinée à la possible rupture d'un isthme ou d'un tombolo, auraient progressivement isolé ces territoires ; l'insularisation aurait ainsi peu à peu limité l'accès à ces villages et compliqué la vie quotidienne de leurs occupants au point de les pousser à s'installer ailleurs sur le Continent.

Se pose également la question de l'impact de la mise en place de formations dunaires sur l'abandon de certains sites de l'Âge du Fer, facteur mis en évidence dans le Trégor par exemple, sur le site de Landrellec à Pleumeur-Bodou (Côtes-d'Armor) où des épisodes successifs (et rapprochés dans le temps) d'ensablement ont contraint les occupants à abandonner l'atelier artisanal (Daire, 2003).

\section{Une vision biaisée des populations de l'Âge du Bronze : un paysage funéraire et rituel/l'importance du relief à l'âge du Bronze}

Les vestiges connus pour l'Âge du Bronze dans ce secteur ne nous permettent d'obtenir qu'une vision partielle de ces populations, puisque les habitats sont méconnus dans l'état actuel des recherches (Fily, 2008 ; Nicolas, 2008). Les deux types de sites principaux mis au jour (tombes et dépôts métalliques) nous laissent entrevoir leurs modes de vie à travers un angle de vue réduit. Seules leur représentation de la mort et leur pratique rituelle de dépôts d'objets en alliage cuivreux aident à appréhender la vie de ces hommes et de ces femmes. Tout un pan de leurs activités quotidiennes reste donc encore à découvrir. Toutefois, l'étude de l'implantation des sites funéraires et des dépôts dans le paysage apporte des informations essentielles sur leur conception de leur environnement.

L'observation de l'implantation des tombes attribuables à l'Âge du Bronze dans le paysage laisse apparaitre que le relief relatif a joué un rôle majeur dans le choix du lieu de dépôt des défunts puisque les zones de hauteur ont nettement été privilégiées dans ce secteur, comme dans le reste du département (Fily, 2008). Les sites funéraires sont en majorité situés en haut des collines, ou très proches de leur sommet. Ils sont également nombreux sur les replats de pente qui représentent par ailleurs plus de la moitié du relief relatif de la zone. Les zones basses, comme les bas de pente, ou les vallées ont à l'inverse été écartées, puisque très peu de sépultures de l'Âge du Bronze ont été retrouvées dans ce 
type de paysage. Les éventuels colluvionnements qui masqueraient les sites ne suffisent pas à justifier une telle absence dans ces zones basses, et ce type de relief devait donc être peu attractif pour y édifier une tombe.

57 Concernant les dépôts métalliques, les zones humides ont assez peu servi de réceptacle pour accueillir cette pratique dans ce plateau littoral, contrairement à certains cours d'eau bretons comme la Vilaine dans laquelle plusieurs objets de l'Âge du Bronze ont été dragués. Les dépôts du secteur sont majoritairement des dépôts terrestres. Les zones de hauteur, et notamment les hauts de pente ont semble t'il été favorisés dans ce département, et tout particulièrement dans le Bas-Léon, où ces types de relief ont souvent été choisis pour enfouir ces objets. Toutefois, de manière générale, leur situation dans le paysage est moins affirmée que pour les sites funéraires, et la prise en compte du relief, bien qu'importante, ne semble pas avoir été le seul élément déterminant de leur implantation. Cette pratique apparaît donc comme plus complexe dans son rapport au paysage que les sépultures.

\section{Quand l'homme façonne le paysage...}

\section{Des structures agraires anciennes}

L'apparition puis le développement de la métallurgie du fer à partir du ve siècle av. n.è. a entraîné une amélioration de l'outillage agricole (socs d'araires, par exemple). Des sillons protohistoriques laissés par des araires ont été fossilisés sous les dunes littorales, à Plougoulm, à Penmarc'h (Finistère), ou encore à Penvenan (Côtes-d'Armor) (Giot et al., 1995).

Or, sur le versant oriental de l'île Guennoc, une zone déclive rejoignant le bord de mer porte des traces de cultures en petites parcelles, courtils ou jardinets qui correspondent à un système apparenté aux " champs celtiques ", et ont pu être en usage tant pendant la Protohistoire qu'au haut Moyen Âge (Giot, 1982 ; Batt et Giot, 1980). Par ailleurs, la partie la plus abritée de l'île Guenoc conservait en outre les reliques d'une flore rappelant une pâture continentale, plus drue, avec trèfle et plantain (Marguerie in Giot, 1987). En outre, des traces de cultures en billons ont été décelées en lumière rasante (photos 7 et 8), tant à Guennoc qu'à Yoc'h, qui correspondent à d'anciennes techniques de labours effectués à la main (Giot et al., 1982) et sont aujourd'hui attribuées à l'Âge du Fer (Daire et Quesnel, 2008). Des traces de cultures assez semblables dans leur organisation et dans leurs dimensions ont d'ailleurs été mises en relation avec l'occupation pré-romaine du site de Hengistbury Head (Dorset, Grande Bretagne) (Lewis, 2002). 
Photo 7 : Traces de cultures en billons et sillons sur le flanc est de l'île Guennoc (d'après un cliché de l'Aéronavale, archives du labo. Archéosciences, Rennes)/Traces of ancient ploughing drills (or furrows) on the eastern slope of Guennoc island (after French Navy, document Archéosciences lab., Rennes)

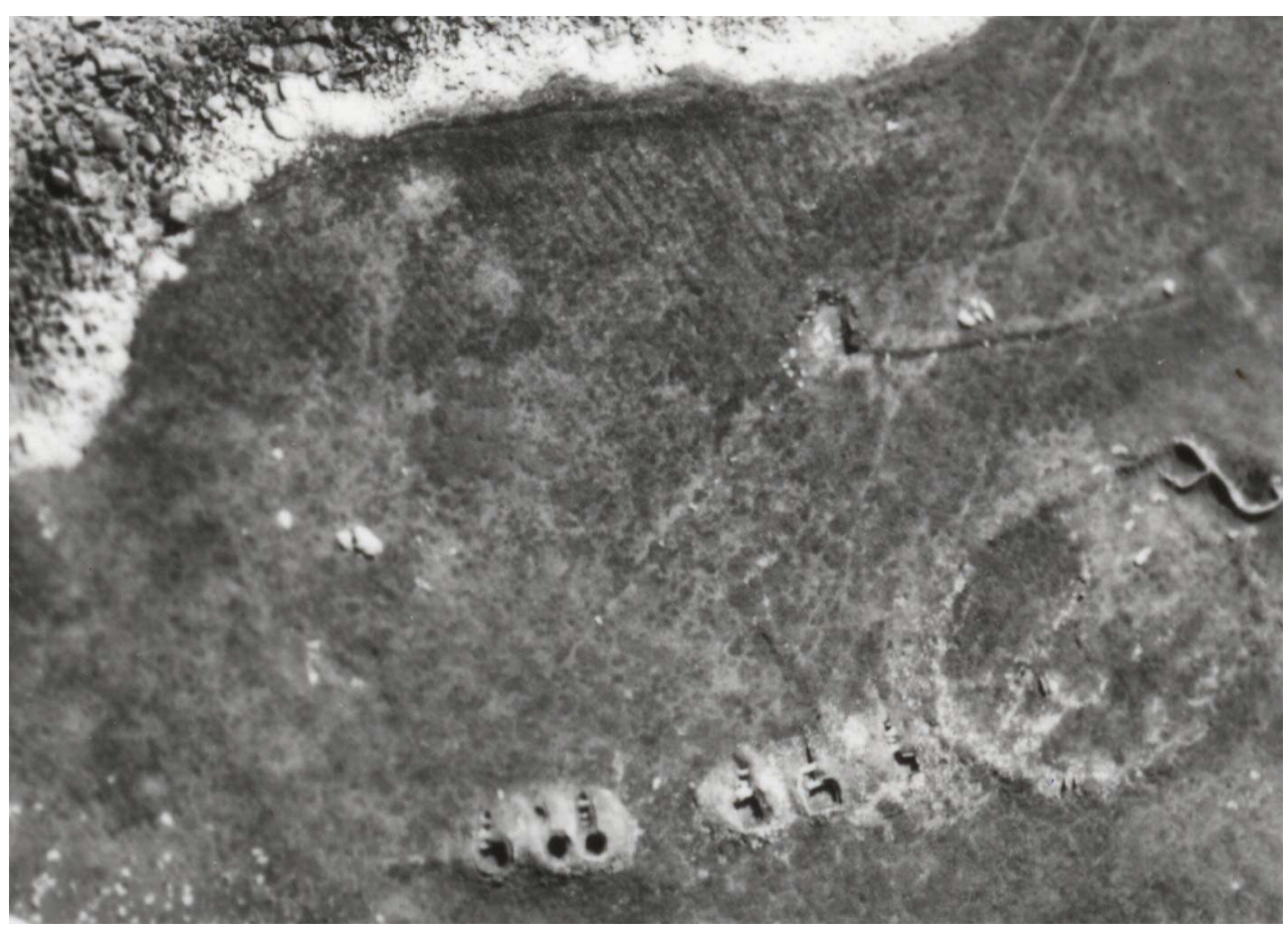

Photo 8 : Traces de cultures en billons et sillons sur le flanc sud-est de l'île d'Yoc'h à Landunvez, à proximité immédiate des installations de goémoniers (d'après un cliché de l'Aéronavale)/Traces of ancient ploughing drills (or furrows) on the south-eastern slope of Yoc'h island (after French Navy, document Archéosciences lab., Rennes)

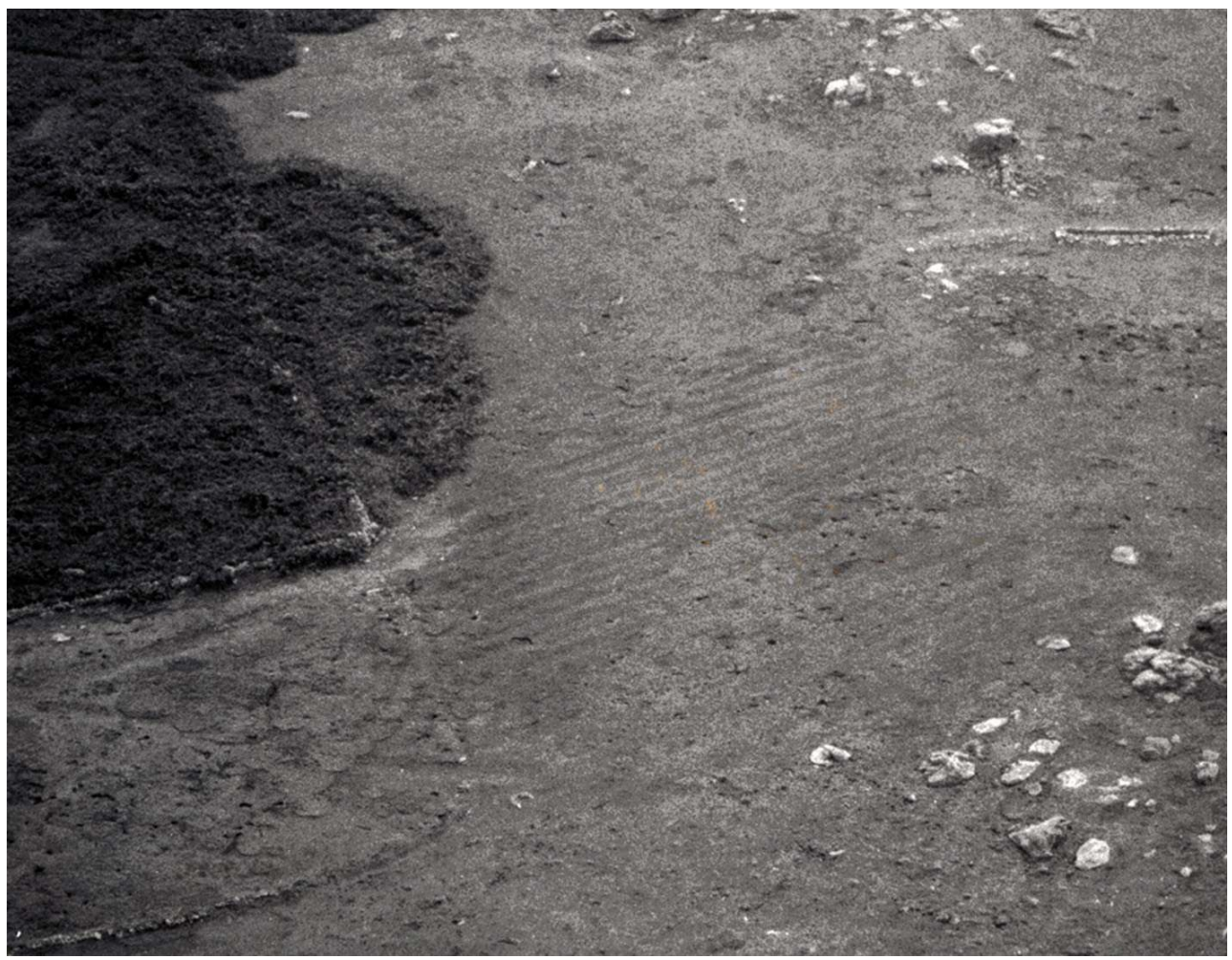


plateau du Bas-Léon révèle d'autres traces d'exploitations agricoles fossilisées, sur l'île Venan à Plouguerneau, en association avec des tombelles dont la datation ne peut être précisée entre l'Âge du Bronze et l'Âge du Fer (archives AMARAI²), de même que sur un vieux sol protégé sous les dunes de Keremma à Tréflez; deux talus, probablement très anciens, émergent sous les dunes de la presqu'île Sainte Marguerite à Plouguerneau et se relient par place aux vieux sols visible, par endroits, sur l'estran (Giot et al., 1982). Les îles et le littoral léonards recèlent un potentiel encore inexploité pour l'étude des anciennes pratiques culturales.

\section{Une structuration du paysage : résilience d'éléments anciens ?}

61 Même s'il est aujourd'hui établi que les structures d'enclos, tels que révélés par les prospections aériennes, peuvent répondre à des finalités différentes (enceintes agricoles, espaces consacrés, cimetières), ils ont en commun de répondre à la fois à une haute fonction symbolique (appropriation d'un espace par sa délimitation) et à des impératifs économiques (confinement du bétail par exemple) ou sociaux, débouchant sur une structuration du paysage.

L'enclos de Kerveatous à Plouarzel (fig. 5) dominant la rive sud de l'Aber Ildut livre un remarquable exemple d'enclos de type protohistorique à multiples fossés concentriques dont le tracé a été partiellement préservé au fil des millénaires dans la structure du paysage bocager, avec une partie du système de talus-fossé intégré dans une limite de champ et respecté par la voirie. Ce site apparait aujourd'hui comme l'un des exemples relativement rares de résilience d'une structure très ancienne, dans ce que nous pouvons considérer comme une relique ponctuelle d'un «bocage organique ». En effet, le secteur étudié montre plusieurs exemples de limites parcellaires très anciennes, associées à des enclos et susceptibles de remonter à l'Antiquité voire à l'Âge du Fer à Plourin, Plouguin et Plouarzel (Daire, 1991, 1993a) mais qui sont en discordance avec le maillage de la structure bocagère ultérieure (Marguerie et al., 2003).

Figure 5 : Enclos paracurviligne à fossés multiples, Kerveatous à Lampaul-Plouarzel, partiellement préservé (à gauche) (cl. M.-Y. Daire) et report sur fonds cadastral (DAO L. Quesnel)/Paracurvilinear enclosure with multiple ditches, Kerveatous in Lampaul-Plouarzel, partly preserved (on the left) (cl. M.-Y. Daire) and drawn on the cadastral map (DAO L. Quesnel)
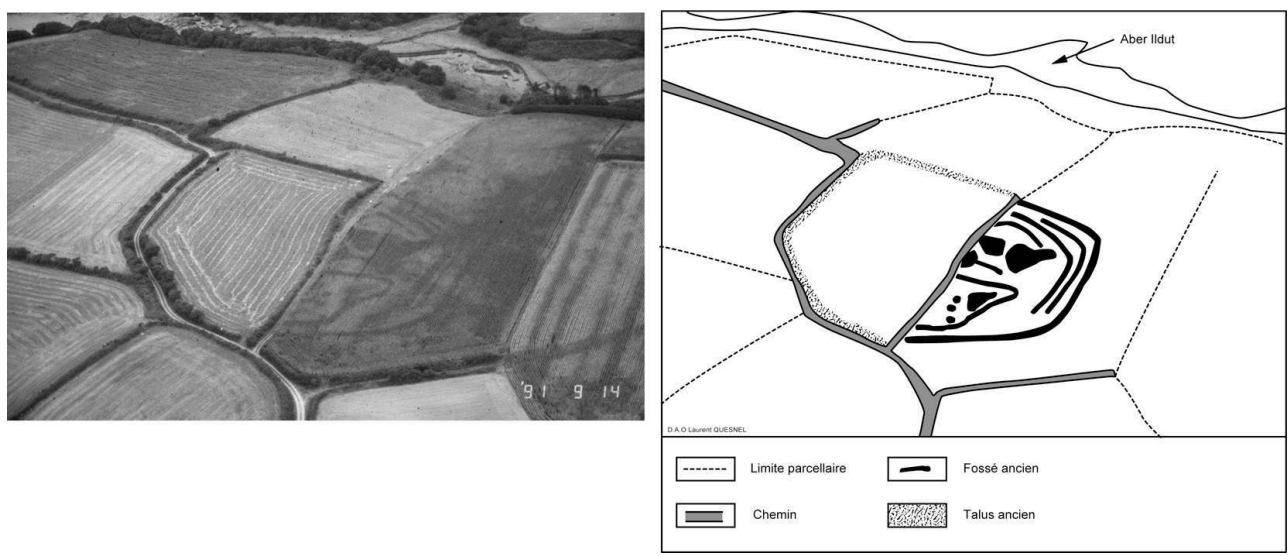

La bande de terre comprise entre les abers Wrac'h et Benoît, correspondant aux communes de Landéda et de Lannilis, comporte des traces assez nombreuses d'occupations protohistoriques et antiques (gisements et sites). Son paysage est marqué 
par la présence de deux voies anciennes, parallèles et orientées est-ouest, qui traversent cette zone en évitant les obstacles naturels que sont les vallées et les reliefs bordant immédiatement les abers (fig. 6); au nord, le chemin $n^{\circ} 1$ est considéré comme un diverticule antique de la voie Kérilien-Pointe Saint-Mathieu; à $1 \mathrm{~km}$ au sud, le chemin $\mathrm{n}$ - 2 forme une limite communale sur $2 \mathrm{~km}$ et présente lui aussi un tracé linéaire. L'analyse des ruptures et continuités de structures parcellaires (à partir des cartes IGN au $1 / 25$ 000), de part et d'autre de ces voies, indique le rôle structurant de ces dernières, sur lesquelles chemins et limites de champs sont largement venus s'appuyer perpendiculairement (ruptures dominantes). Au sein de cet ensemble, l'analyse parcellaire de l'environnement immédiat de l'enclos protohistorique ou antique découvert par prospection aérienne à Bon Plaisir (Daire, 1991) suggère une organisation dont la trame suit les mêmes orientations selon les axes N-S et E-W, l'enclos étant luimême en bonne concordance avec cette organisation (fig. 7). Il s'agit là de tendances suggérant une forme de "planification agraire", demandant à être vérifiées et approfondies par des méthodes de filtrage mathématique ou physique appliquées à l'analyse du paysage.

Sur le littoral de Plouguerneau, de l'autre côté de l'aber Wrac'h, le nombre de vieux chemins menant à la côte (pour n'aboutir aujourd'hui nulle part) a été attribué à la desserte de territoires aujourd'hui érodés ou disparus (Giot et al., 1982) ; les variations très inégales du trait de côte sont effectivement une complication supplémentaire dans les tentatives de lecture des paysages littoraux.

Figure 6 : Cartographie simplifiée des chemins organisés selon les axes des voies anciennes et des sites archéologiques du secteur de Landéda et de Lannilis (d'après Daire, 1991, DAO L. Quesnel)/ Simplified mapping of the paths organised according ancient roads and of the archaeological sites in the Landéda and Lannilis area (after Daire, 1991, DAO L. Quesnel)

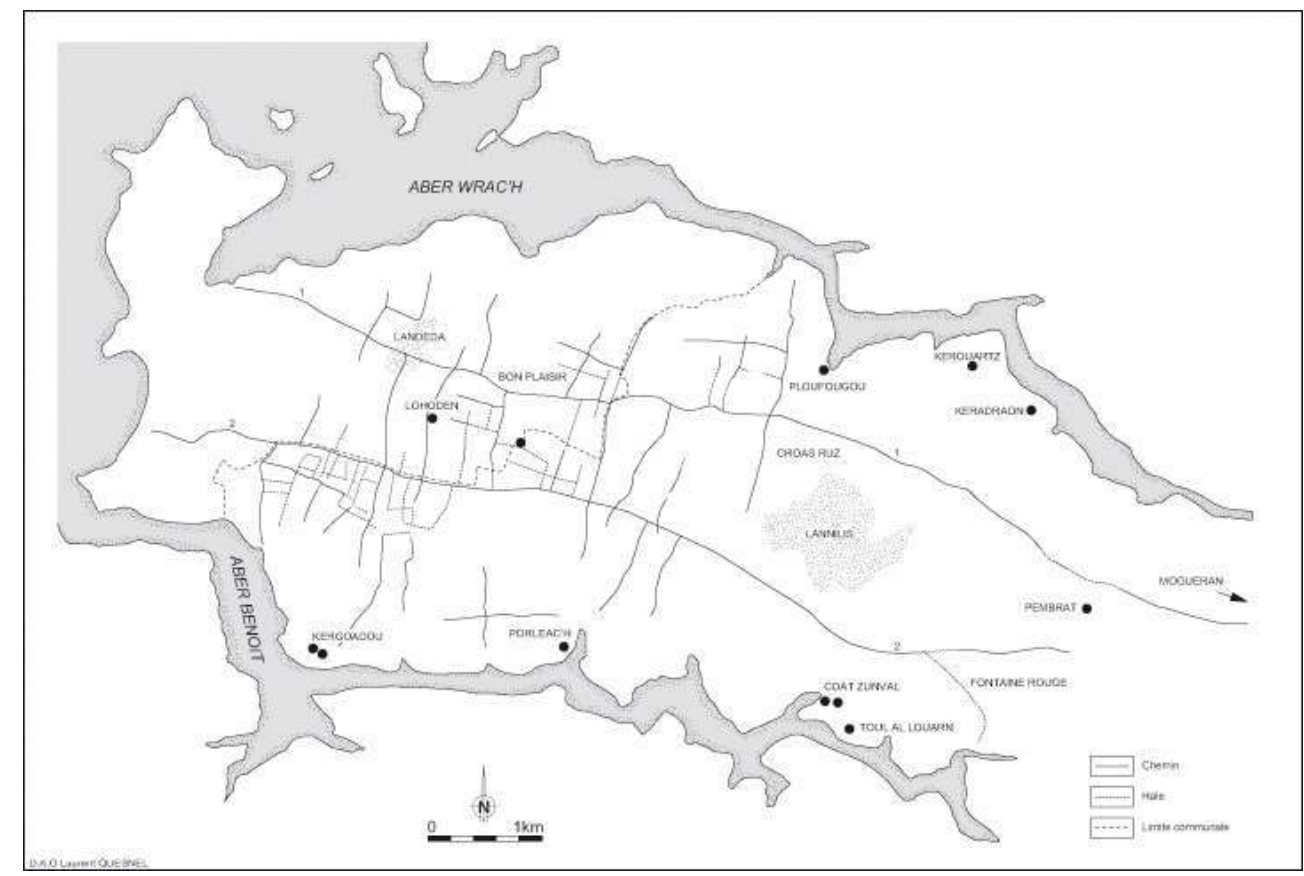




\section{Une gestion des ressources?} de fournir une matière première abondante. Dans ces formations secondaires, la clarté du couvert permet, dans un premier temps, une croissance des arbres plus rapide. L'analyse de la largeur des cernes de croissance sur les charbons de chêne montre nettement une augmentation de l'épaisseur des cernes entre le Néolithique et le second Âge du Fer, interprétée comme la conséquence d'une moindre compétition interindividuelle entre les arbres et donc une ouverture du milieu forestier (Marguerie, Hunot, 2007) (fig. 2A).

Figure 7 : Schéma cadastral de l'environnement de l'enclos détecté à Bon Plaisir, Landéda (d'après Daire, 1991, DAO L. Quesnel)/Cadastral scheme of the surrounding area of the enclosure at Bon Plaisir, Landéda (after Daire, 1991, DAO L. Quesnel)

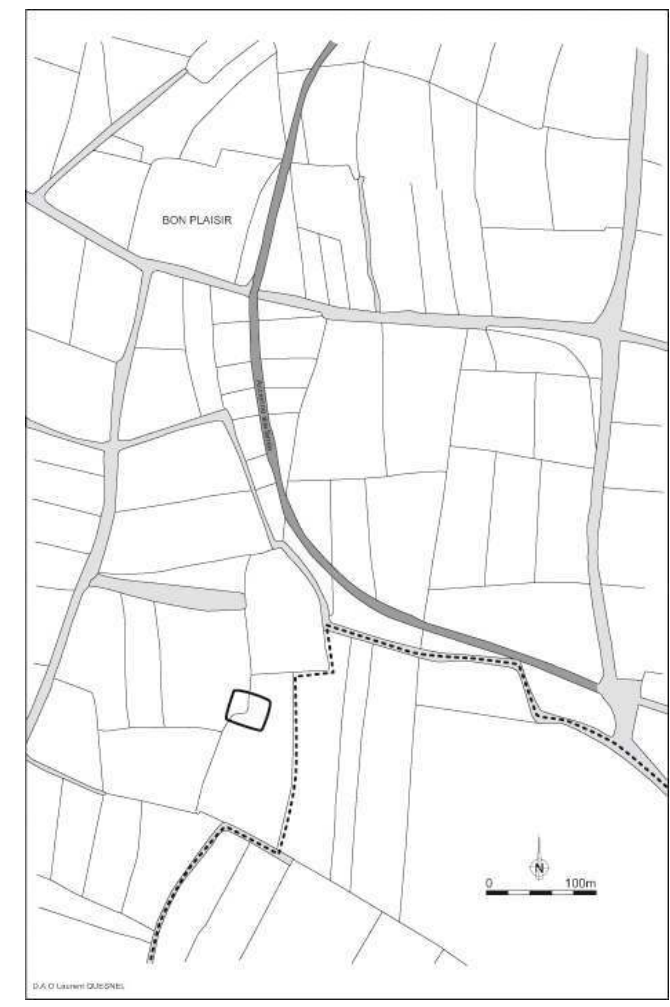

67 Cette exploitation des forêts et la dégradation progressive des sols ainsi mis à nu sont à l'origine des landes ou fourrés régressifs armoricains, formations végétales largement exploitées par l'homme à l'Âge du Fer si l'on en croit les taxons attestés par l'étude des 
charbons de bois issus des foyers domestiques, notamment ; c'est à l'époque de La Tène que le développement de la lande devient le plus sensible, en corollaire de l'ouverture du milieu forestier (Marguerie, 1995). Après la conquête romaine, le processus d'exploitation de la forêt a pris, dans certains secteurs géographiques, un caractère systématique (Marguerie, 1992).

Les pratiques cynégétiques ne se manifestent que timidement dans les ensembles du Finistère nord. Pour le Bronze final, la présence de restes de sanglier, espèce inféodée aux forêts de feuillus et aux forêts mixtes, peut toutefois être mentionnée en association avec le dépôt en langue de carpe de Kerc'hleus à Saint-Pabu (Giot et al., 1995).

Les analyses anthracologiques réalisées sur les charbons de bois provenant du hameau de l'île d'Yoc'h montrent la présence d'espèces héliophiles susceptibles d'être rencontrées dans les landes, espèces utilisées préférentiellement pour le fonctionnement des foyers domestiques et/ou artisanaux, tandis que de grosses branches ou des troncs de chêne ont été plutôt sélectionnées comme bois d'œuvre (Marguerie, 1992), témoignant ainsi d'une gestion raisonnée des ressources ligneuses et végétales.

Dans cette gestion du milieu naturel, la question se pose de l'impact de l'activité des bouilleurs de sel gaulois sur l'environnement végétal. Même si les inventaires archéologiques montrent, dans le nord du département du Finistère, une densité d'ateliers moindre à celle d'autres secteurs géographiques, cette activité salicole reconnue à l'île d'Yoc'h, à Beg ar Vir, à l'île du Bec ou encore sur l'île de Triélen dans l'archipel de Molène, et qui s'est considérablement développée au cours des deux derniers siècles av. n.è. est hautement consommatrice en combustible. Corroborant les résultats des analyses paléoenvironnementales, les expérimentations menées dans la région montrent que ces grands fours à sel fonctionnaient fort bien avec des combustibles de type fagots ou végétation de landes; l'utilisation des algues marines est également attestée, de manière indirecte, par la présence de petits coquillages «parasites » qui y sont restés accrochés (com. pers. C. Dupont) (Daire et al., à paraître). L'impact de telles activités sur le milieu a peut-être été minimisé du fait que, pendant très longtemps, les occupations protohistoriques sur le littoral ont été considérées comme " provisoires », et l'évocation de " campements côtiers temporaires ", voire éphémères, donc implicitement sans grand effet sur l'environnement, ponctue encore une littérature pas si ancienne. Les plus récents réexamens de cette question montrent que la plupart de ces établissements littoraux, stables, structurés, ont connu une durée de fréquentation de plusieurs générations humaines sur les mêmes lieux, de la même manière que certains habitats de l'intérieur des terres (Daire, 2003). Les données paléobotaniques concernant les pratiques agricoles montrent d'ailleurs qu'il n'y a plus lieu d'opposer une situation côtière et continentale à l'Âge du Fer (Marguerie, 1995 ; Gaudin, 2004).

71 Les peuplements de l'Âge du Fer dans ce secteur ont largement exploité les ressources minérales diversifiées et accessibles du rebord de plateau, tant dans une architecture domestique et artisanale faisant largement intervenir des murs en pierre sèche que dans des pratiques funéraires; la densité des stèles funéraires de l'Âge du Fer dans la bande côtière est de ce point de vue très significative (Daire, 2005) ; dans le détail, divers modes de gestion des ressources minérales du secteur sont perceptibles. Si les bâtisseurs gaulois de Guennoc ou de l'île d'Yoc'h ont assez "sauvagement» pillé les monuments mégalithiques de leurs prédécesseurs du Néolithique pour construire leurs propres maisons, certains comportements témoignent de pratiques moins opportunistes. Ainsi, en revenant aux centaines de stèles funéraires de l'Âge du Fer présentes dans ce secteur 
géographique, les analyses pétrographiques montrent que certains massifs granitiques, comme celui de Saint-Renan ou encore celui de Tréganna, ont été largement privilégiés par rapport à d'autres, pour des raisons liées aux qualités mécaniques des pierres concernées, et cela indépendamment de la surface et du potentiel d'exploitation des gisements (Chauris, 1995 ; Daire, 2005).

Dans ces conditions, il conviendra de s'interroger plus avant sur l'impact de ces occupations humaines sur l'environnement côtier au sens large, sur l'environnement végétal, bien sûr mais aussi sur les ressources minérales ou encore animales.

\section{Discussion}

L'intérêt d'une approche intégrant l'analyse géographique des sites côtiers est à la fois d'apporter un éclairage sur l'interprétation fonctionnelle des occupations et, par balancement, d'asseoir les reconstitutions paléogéographiques en s'appuyant sur l'apport des vestiges archéologiques. Cependant, la démarche synthétique conduite ici conservera malheureusement un certain nombre de limites; certaines sont liées à l'histoire environnementale de cette côte: "Que ce soit devant Ploudalmézeau, LampaulPloudalmézeau, Saint-Pabu, Landéda, Lillia, Plouguerneau, Guissény ou Kerlouan, par exemple, et plus à l'est encore, les indices sont nombreux sur les terroirs progressivement ennoyés. Il est important de bien se rendre compte qu'on ne saurait raisonner au sujet du peuplement de ces territoires à partir des aspects et du trait de côte actuels, même pour un recul de quelques siècles dans le temps » (Batt et Giot, 1980).

Nous avons souligné, en préambule, d'autres limites inhérentes à l'état de la documentation disponible sur le secteur considéré. Si les données concernant d'une part l'Âge du Bronze et, d'autre part, l'Âge du Fer (fig. 1 B et C) paraissent globalement comparables et quantitativement suffisantes pour tenter une analyse plus approfondie en terme d'évolution, des décalages très importants sont à souligner: les données disponibles pour l'Âge du Bronze concernent principalement le domaine funéraire et les dépôts d'objets, mais les habitats et les zones de travail demeurent méconnus pour l'instant. Une analyse détaillée de l'évolution des conditions d'implantation des habitats sur l'ensemble de la Protohistoire récente n'est donc pas possible à ce stade. Dans le domaine funéraire, la situation est plus équilibrée entre les deux périodes d'un point de vue quantitatif ; mais, alors que les tumulus de l'Âge du Bronze ne posent aucun problème de géoréférencement, la localisation des stèles funéraires des ve-Ive siècles avant n.è., connues par centaines dans le Finistère, est entachée d'une grande imprécision: ces stèles ne se trouvent jamais à leur emplacement d'origine et peuvent avoir subi des déplacements allant de quelques centaines de mètres à plusieurs kilomètres, voire davantage dans certains cas. Ceci explique que les analyses spatiales sont forcément segmentées d'un point de vue chronologique, tant que l'on ne disposera pas d'une documentation plus homogène.

Néanmoins, les éléments de synthèse proposés ici permettent d'élaborer à grands traits un schéma de l'évolution chronologique, spatiale et culturelle des peuplements continentaux du Bas-Léon, en lien avec l'évolution du milieu naturel, schéma qu'il conviendra de vérifier et de valider par des investigations futures.

76 Au cours des premières phases de l'Âge du Bronze, le peuplement de ce territoire est certainement conditionné par un environnement largement dominé par le milieu 
forestier, y compris sur la bande côtière. À partir du début du Bronze moyen (vers 1600 av. n.è.), l'ouverture du paysage en relation avec le développement des pratiques culturales va sans doute de pair avec le développement d'enclos ruraux à vocation agricole, dont l'existence est attestée depuis peu dans l'ouest de la France par la récente fouille de Lamballe (Côtes-d'Armor) (Ménez et Hinguant, 2010). Des investigations plus approfondies sur certains des nombreux enclos protohistoriques découverts dans le Léon par prospection aérienne (Daire 1991, 1993a, 1993b) pourraient venir confirmer cette hypothèse.

77 Mais ces anciens peuplements sont également conditionnés par les ressources naturelles disponibles. À ce titre, il paraît probable que le peuplement datant de la période de l'Âge du Bronze, considérée dans son ensemble, ait été pour une part conditionné, dans le BasLéon, par la présence et l'exploitation de gisements d'étain (Saint-Renan, Bourg-Blanc), d'autant plus intéressants en terme de circuits commerciaux qu'ils se trouvent en bordure relative de côte ; en cela, le rôle de la vallée de l'aber Ildut avec sa richesse en alluvions stannifères et son rôle de vecteur vers la mer sont certainement des éléments à prendre en compte.

$78 \mathrm{Si}$, pour cette période, les sites d'habitats étudiés font encore défaut, l'implantation des nombreux tumulus de la région révèle une prédilection pour les points topographiques relativement hauts pendant l'Âge du Bronze ancien et moyen (entre 2200 et 1300 av. n.è.). Outre Manche, cette préférence pour édifier les sites funéraires dans les zones de hauteur a également été soulignée (Tilley, 1995, 1996 ; Woodward et Woodward, 1996). Il semblerait que ces points hauts de la topographie aient été choisis pour permettre aux morts de dominer le paysage environnant, et de marquer, ou peut-être jalonner visuellement par un tertre, un territoire. Par ailleurs, le panorama visible depuis ces monuments est souvent impressionnant de par son étendue et apparait grandiose aux yeux d'un observateur situé près de la tombe.

79 Si les caractéristiques majeures du peuplement des premières phases de l'Âge du Fer, encore assez peu documentées, s'inscrivent dans la continuité de la période précédente, un tournant semble se marquer au cours du second Âge du Fer (450-50 av. n.è.), en lien avec un essor démographique bien attesté dans l'Ouest de la France (Giot et al., 1995). Celui-ci se manifeste notamment par le développement des enclos à vocation agricole et des sites fortifiés mais aussi par des fréquentations humaines plus nombreuses sur la côte, en lien avec l'exploitation des ressources marines (production de sel, pêche) et les possibilités de relations commerciales à moyenne ou longue distance (amphores vinaires d'Italie, par exemple). Pour cette période, on peut émettre l'hypothèse que la structuration du territoire anthropisé est, semble-t-il, autant conditionnée par les contraintes paysagères que par la structure sociale : avec toute la prudence qui s'impose compte tenu des limites de l'échantillonnage de sites sur ce secteur de l'aber Ildut (cf. supra), cette première analyse pose toutefois la question de la distribution des habitats en fonction de divisions naturelles du paysage qui, pour des secteurs géographiques comparables du sud de l'Angleterre, ont été reliées à une répartition des territoires entre zones cultivées et terres de pâture (Palmer, 1984).

\section{Conclusions}

80 Le milieu littoral se prête particulièrement bien au développement d'approches géoarchéologiques ou archéogéographiques et favorise les démarches interdisciplinaires 
« propices à l'étude des relations réciproques homme/milieu, parce que les traces y sont accusées à l'extrême et leur enregistrement en est d'autant facilité » (Burnouf et al., 1996). La présente synthèse avait pour principale finalité la mise en évidence d'un potentiel de recherche énorme dans le secteur géographique du Bas-Léon, potentiel aussi riche pour les phases récentes de la Protohistoire que pour la période Néolithique (Sparfel et Pailler [dir.], 2009), mais ce potentiel est encore sous-exploité dans le domaine côtier continental ; des projets transdisciplinaires à venir pourraient s'inspirer des travaux déjà réalisés et publiés pour l'île d'Ouessant (Le Bihan et Villard, 2007, 2010) ou encore de ceux bien avancés pour l'archipel de Molène (Pailler et al., ce volume); les premiers objectifs seraient d'affiner les données morphologiques et chronologiques sur les sites domestiques et funéraires (sites enclos et habitats ouverts) du Ier millénaire av. n.è., mais également d'identifier formellement les réseaux d'échanges locaux à partir de matériaux tels que la céramique et de développer des séries d'analyses paléoenvironnementales.

81 La synthèse présentée ici aboutit à un schéma chronologique, spatial et culturel, pour le Ier millénaire av. n.è., montrant deux phases nettement différenciées :

82 - une première phase où, au sein d'un paysage encore largement boisé, des habitats dispersés et probablement assez peu nombreux appartiennent à une société encore fortement hiérarchisée qui exploite notamment les ressources métallurgiques du secteur;

83 - une seconde phase marquée par un peuplement au maillage très dense, composé à la fois d'agriculteurs et d'éleveurs tirant encore des ressources complémentaires des massifs forestiers discontinus qui les entourent, mais aussi de communautés d'artisans de plus en plus spécialisés qui s'installent en nombre croissant sur la côte, afin d'en exploiter les ressources naturelles (sel, pêche, coquillages, etc.) et d'y développer des réseaux d'échanges à moyenne et longue distance (amphores vinaires de Méditerranée, par exemple). Au-delà de ces traits généraux que nous sommes maintenant en mesure de brosser pour caractériser les interactions homme/milieu dans le Bas-Léon, des investigations complémentaires seront nécessaires pour affiner notre vision des occupations et des activités humaines en lien avec l'évolution du milieu naturel au cours de la Protohistoire récente, évolution qui se révèle particulièrement complexe en milieu littoral.

84 Les auteurs tiennent à exprimer leur gratitude au coordonnateur du volume, pour sa bienveillante patience, ainsi que les deux relecteurs de cet article, pour leurs critiques très constructives et leurs suggestions pertinentes.

\section{BIBLIOGRAPHIE}

ARBOUSSE-BASTIDE T., 2000. Une approche des enclos du nord de la Bretagne Analyse et comparaison des sites protohistoriques, Oxford, British Archaeological Reports, S885, 89 p., 63 fig.

BATT M., GIOT P.-R., 1980. Quelques observations d'archéologie du paysage en Finistère, Bulletin de la Société Archéologique du Finistère, tome CVIII, p. 17-25. 
BAUDRY A., 2005. Approvisionnement et alimentation carnée sur les sites de l'âge du Fer en Bretagne et en Normandie, première approche. L'exemple du site de « La Campagne » à Basly, Revue Archéologique de l'Ouest, n²2, p. 165-180.

BRIARD J., ONNÉE Y., 1996. L'Âge du Bronze à l'île Guennoc (Landéda, Finistère), Bulletin de l'AMARAI (Association Manche Atlantique pour la Recherche Archéologique dans les Îles), n 9, p. 31-42.

BRIGAND L., 2002. Les îles du Ponant. Histoire et géographie des îles et archipels de la Manche et de l'Atlantique, Quimper, Palantines, $479 \mathrm{p}$.

BURNOUF J., GARMY P., TASTET J.-P., 1996. Paléo-paysages littoraux et occupations du sol holocènes en Nord-Médoc (Gironde, France), dans GUILLEMET D., PÉRET J. (dir.), Les sociétés littorales du Centre-Ouest atlantique, de la Préhistoire à nos jours, Mémoires de la Société des Antiquaires de l'Ouest et des Musées de Poitiers, $5^{\mathrm{e}}$ série, tome IV, p. 39-49.

CHAURIS L., 1995. Nature lithologique des stèles de l'Âge du Fer dans l'extrémité orientale du pays de Léon, Bulletin de l'AMARAI (Association Manche Atlantique pour la Recherche Archéologique dans les Îles), $\mathrm{n}^{\circ} 8, \mathrm{p}$. 57-84.

CHOUQUER G., 2003. Objets en crise, objets recomposés, Études Rurales, p. 167-68.

DAIRE M.-Y., 1991. Première campagne de prospection aérienne intensive en Léon, Bulletin de la Société Archéologique du Finistère, tome CXX, p. 105-132.

-, 1993a. Une nouvelle campagne de prospection aérienne en Léon, Bulletin de la Société Archéologique du Finistère, tome CXXII, p. 137-161.

-, 1993b. Photographie aérienne à basse et/ou haute altitudes : exemples d'apports méthodologiques en Bretagne, Revue d'Archéométrie, $\mathrm{n}^{\circ}$ 17, p. 11-25.

-, 2001. Un hameau armoricain de la fin de l'Âge du Fer sur l'île d'Yoc'h, dans collis J. (dir.) : Society and environment in Iron Age Europe, Actes du XVIII ${ }^{\mathrm{e}}$ Colloque de l'AFEAF, Winchester, april 1994, éd. J. Collis, Université de Sheffield, p. 159-189.

-, 2003. Le sel des Gaulois, Ed. Errance, Paris, 152 p.

-, 2005. - Les stèles de l'Âge du Fer dans l'Ouest de la Gaule. Réflexions sur le monde des morts et le monde des vivants, Rennes, Les Dossiers du CeRAA (Centre Régional d'Archéologie d'Alet), n AB, 172 p.

-, 2008. Des pêcheries d'estran sur les côtes du Bas Léon, dans DAIRE M.-Y., LANGouËT L., (dir.), Les pêcheries de Bretagne. Archéologie et Histoire des pêcheries d'estran, Rennes, AMARAI ( Association Manche Atlantique pour la Recherche Archéologique dans les Îles)/CeRAA (Centre Régional d'Archéologie d'Alet), p. 113-122.

DAIRE M.-Y., BIZIEN-JAGLIN C., BAUDRY A., à paraître. An archaeology of coastal salt industry: the inescapable part of experiments, dans GHEORGHIU D., WIEKEN J., ZIMMERMAN E., Experimenting the past. The position of experimental archaeology in the archaeological paradigm of the $21^{\text {st }}$ century. Actes du 14 Congrès International de l'EAA (European Association of Archaeologists), Malte, sept. 2008. DAIRE M.-Y., QUESNEL L., 2008. Des Gaulois sur l'île Guennoc (Landéda, Finistère), Revue Archéologique de l'Ouest, $\mathrm{n}^{\circ} 25$, p. 93-138.

DU CHATELLIER P., 1891. De quelques cachettes découvertes dans le Finistère, L'Anthropologie, $\mathrm{n}^{\circ}$ 2, p. 17-22.

-, 1899. Le Bronze dans le Finistère, Bulletin de la Société archéologique du Finistère, tome XXVI, p. 18 et p. 250-301.

ÉLEGOËT L., 2007. Le Léon. Histoire et géographie contemporaine, Quimper, Palantines, 293 p. 
FILY M., 2008. Les monuments funéraires et les dépôts métalliques dans le paysage rituel de l'Âge du Bronze : l'exemple du centre-ouest de la Bretagne et du Finistère littoral (France), thèse de doctorat "Archéologie et Archéosciences », Université de Rennes 1, 621 p. et un Cédérom.

FITZPATRICK S. M., ANDERSON A., 2008. Islands of isolation: archaeology and the power of aquatic perimeters, Journal of Island and Coastal Archaeology, $\mathrm{n}^{\circ} 3$, p. 4-16.

GALliou P., 2010. Le Finistère, Paris, (2 ${ }^{\text {nde }}$ éd.), Acad. Inscriptions et Belles Lettres, coll. « Carte Archéologique de la Gaule », 494 p.

GAUDIN L., 2001. L'évolution d'un trait côtier, exemple du rivage de la région du Golfe du Morbihan, Bulletin de l'AMARAI (Association Manche Atlantique pour la Recherche Archéologique dans les Îles), n 14, p. 5-14.

-, 2004. Transformations spatio-temporelles de la végétation du nord-ouest de la France depuis la fin de la dernière glaciation. Reconstitutions paléo-paysagères, thèse de doctorat « Archéologie et Archéosciences ", Université de Rennes 1, 763 p.

GEBHARDT A., 1989. Île d'Yoc'h en Landunvez (Finistère), rapport d'étude sédimentologique, Rapport reprographié, UPR 403 du CNRS, Rennes, 13 p.

GIOT P.-R., 1982. Enez Guennoc ou Geignog, un ancien microcosme celtique, dans Mélanges d'archéologie et d'histoire médiévale offerts à M. de Boüard, Paris, Société de l'École des Chartes éd., coll. « Mémoires et Documents ; 27 », p. 179-190.

-, 1987. Barnenez, Carn, Guennoc, éd. Travaux du Laboratoire d'Anthropologie de l'Université de Rennes 1, Rennes, 2 vol., 212 p. et 57 pl.

,- 1990. Le niveau de la mer : changeant, fluctuant, mouvant, Bulletin de l'AMARAI (Association Manche Atlantique pour la Recherche Archéologique dans les Îles), n 3, p. 5-16.

GIOT P.-R., BATT M., MORZADEC M.-T., 1982. Archéologie du paysage agraire armoricain, éd. Travaux du Laboratoire d'Anthropologie de l'Université de Rennes 1, Rennes, 76 p.

GIOT P.-R., BRIARD J., PAPE L., 1995. Protohistoire de la Bretagne, Rennes, Ouest-France, 423 p.

GIOT P.-R., COGNÉ J., 1951. L'Âge du Bronze ancien en Bretagne, L’Anthropologie, tome 53, p. 413-432.

GUILCHER A., HALLÉGOUËT B., 1991. Coastal dunes in Brittany and their management. Journal of Coastal Research, vol. 7, $\mathrm{n}^{\circ}$ 2, p. 517-533.

HALLÉGOUËT B., 1971. Le Bas-Léon (Finistère), étude géomorphologique, thèse de $3^{\mathrm{e}}$ cycle, Université de Bretagne Occidentale (UBO), Brest.

-, 1978. L'évolution des massifs dunaires du pays de Léon, Penn ar Bed, vol. 11, n 95, p. 417-430.

HALLÉGOUËT B., 1981. Les crêtes littorales dunifiées du massif armoricain : formation et évolution, Géographie physique et Quaternaire, vol. 35, n² 2, p. 205-218.

LE PENNEC S. 2007. Le réseau routier antique du Léon, dans ELEGOËT L., Le Léon. Histoire et géographie contemporaine. Quimper, éd. Palantines, p. 40-41.

LE BIHAN J.-P., VILLARD J.-F., 2007. Archéologie d'une île à la pointe de l'Europe : Ouessant - Tome 1 : le site archéologique de Mez-Notariou et le village du premier âge du Fer. Centre Archéologique du Finistère éd., Quimper, 351 p.

-, 2010. Archéologie d'une île à la pointe de l'Europe : Ouessant, Tome 2 : l'Âge du Bronze, Quimper, Centre Archéologique du Finistère éd., 588 p. 
LEWIS H., 2002. An investigation of ancient cultivation remains at Hengistbury Head, Site 6, Christchurch, Dorset. Proceedings of the Prehistoric Society, $n^{\circ}$ 68, p. 83-102.

MARGUERIE D., 1990. L'environnement à l'Âge du Fer en Bretagne, dans Les gaulois d'Armorique. Actes du XII ${ }^{\mathrm{e}}$ colloque de l'AFEAF (Association française d'Étude de l'Âge du Fer), éd. Revue Archéologique de l'Ouest, suppl. n³, p. 115-121.

-,1992. Évolution de la végétation sous l'impact humain en Armorique du Néolithique aux périodes historiques, Rennes, Travaux du Laboratoire d'Anthropologie de l'Université de Rennes $1, \mathrm{n}^{\circ} 40$, $313 \mathrm{p}$.

-, 1995. L'environnement anthropisé, dans GIOT P.-R., BRIARD J., PAPE L. (dir.), Protohitoire de la Bretagne, Rennes, Ouest France, p. 19-24.

MARGUERIE D., ANTOINE A., THENAIL C., BAUDRY J., BERNARD V., BUREL F., CATTEDDU I., DAIRE M.-Y., GAUTIER M., GEBHARDT A., GUIBAL F., KERGREIS S., LANOS P., LECOEUR D., LE DU L., MEROT P., NAAS P., OUIN A., PICHOT D., VISSET L., 2003. Bocages armoricains et sociétés, genèse, évolution et interactions, dans Des milieux et des hommes : fragments d'histoires croisées, Elsevier éd., p. 115-131.

MARGUERIE D., HUNOT J.-Y., 2007. Charcoal analysis and dendrology: data from archaeological sites in western France, Journal of Archaeological Sciences, n³4, 1417-1433.

MÉNEZ Y., HINGUANT S., 2010. Fouilles et découvertes en Bretagne, Rennes, Ouest-France/INRAP, coll. « Histoire », 144 p.

MORZADEC-KERFOURN M.-T., 1974. Variation de la ligne de rivage armoricaine au Quaternaire, analyses polliniques de dépôts organiques littoraux. Rennes, Mémoires de la Société Géologique et Minéralogique de Bretagne, $\mathrm{n}^{\circ} 17,208 \mathrm{p}$.

-, 1995. L'environnement naturel, dans GIOT P.-R., BRIARD J., PAPE L., Protohitoire de la Bretagne, Rennes, Ouest France, p. 18-19.

NICOLAS C., 2008. Les pointes de flèches armoricaines du nord du Finistère. Étude typologique et technologique d'un bien socialement valorisé de l'Âge du Bronze ancien, Mémoire de Master 1 d'Archéologie, Université de Paris 1, 148 p.

PAILLER Y. et al., 2011. Évolution des paysages et occupation humaine en mer d'Iroise (Finistère, Bretagne) du Néolithique à l'Âge du Bronze, Norois, ce volume, p. 39-68.

PAPE L., 1978. La Civitas des Osismes à l'époque gallo-romaine, Kliencksieck éd., Paris, 245 p.

PALMER R., 1984. Danebury (an Iron Age hillfort in Hampshire), Vol. 3 : An aerial photographic interpretation of its environs, Royal Commission of Historical Monuments Editions, suppl. series 6, $133 \mathrm{p}$.

SPARFEL Y., PAILLER Y. (dir.), 2009. Les mégalithes de l'arrondissement de Brest, Coll. Patrimoine Archéologique de Bretagne, co-éd. CeRAA (Centre Régional d'Archéologie d'Alet) et ICB (Institut Culturel de Bretagne), Rennes, 290 p.

STEPHAN P., 2008. Les flèches de galets de Bretagne : morphodynamiques passée, présente et prévisible, Thèse de doctorat en géographie physique, Université de Bretagne Occidentale, Brest, 558 p.

,- 2011 . Colmatage sédimentaire des marais maritimes et variations relatives du niveau marin au cours des 6000 dernières années en rade de Brest (Finistère), Norois, ce volume, p. 9-37.

STÉPHAN P., SUANEZ S., FICHAUT B., 2009. Variations holocènes du niveau marin en Bretagne occidentale, dans SPARFEL Y., PAILLER Y. (dir.), Les mégalithes de l'arrondissement de Brest, Rennes, 
CeRAA (Centre Régional d'Archéologie d'Alet)/ICB (Institut Culturel de Bretagne), coll.

« Patrimoine Archéologique de Bretagne », p. 10-11.

TILLEY C., 1995. Rocks as Resources : Landscapes and Power, Cornish Archaeology, n 34, p. 5-57.

,- 1996. The Power of Rocks : Topography and Monumental Construction on Bodmin Moor, World archaeology, tome $28, \mathrm{n}^{\circ} 2$, p. 161-176.

WOODWARD A. B , WOODWARD P. J., 1996. The Topography of some Barrow Cemeteries in Bronze Age Wessex, Proceedings of the Prehistoric Society, tome 62, p. 275-291.

\section{NOTES}

1. Le site de Mez Notariou à Ouessant est fouillé par J.-P. Le Bihan (Centre Archéologique du Finistère) depuis une vingtaine d'années.

2. Base de données de l'AMARAI: Association Manche Atlantique pour la recherche archéologique dans les îles, Laboratoire Archéosciences, Université de Rennes 1.

\section{RÉSUMÉS}

Les auteurs procèdent ici au réexamen des données concernant les peuplements et les paysages du littoral du Bas-Léon (Finistère), au cours du Ier millénaire avant notre ère. L'analyse des abondants résultats issus d'approches multidisciplinaires permet de dégager des indicateurs d'évolutions sociales et environnementales, suggérant la mise en évidence de deux phases nettement différenciées au cours de la période. Cette présentation conduit à esquisser un schéma d'évolution chronologique, spatiale et culturelle des peuplements et des paysages ; au-delà de certaines limites, cette synthèse permet de proposer un nouvel éclairage sur des dynamiques et des processus encore peu abordés jusqu'ici dans la région et susceptibles d'être approfondis à travers de nouvelles démarches géoarchéologiques.

The authors provide here the review of data on the ancient populations and the coastal landscape of Bas-Léon (Finistère), during the 1st millennium BC. The analysis of the abundant results from multidisciplinary approaches can provide indicators of environmental and social developments, suggesting the identification of two clearly distinct phases during the period. This presentation leads to sketch an outline of the chronological, spatial and cultural evolution considering populations and landscapes; beyond certain limits, this synthesis allows proposing a new light on the dynamics and processes still little addressed so far in the region and which could be deepened through new geoarchaeological approaches.

\section{INDEX}

Mots-clés : géoarchéologie, société, paysage, littoral, ressource, mer

Keywords : geoarchaeology, society, landscape, coast, resource, sea 


\section{AUTEURS}

\section{MARIE-YVANE DAIRE}

UMR 6566 CReAAH Centre de Recherche en Archéologie, Archéosciences, Histoire (CNRS, Universités de Rennes 1, Rennes 2, Le Mans, Nantes, ministère de la Culture, et INRAP), Laboratoire Archéosciences, Bâtiment 24-25, Université de Rennes 1, Campus de Beaulieu 35 042, Rennes cedex, France marie-yvane.daire@univ-rennes1.fr

\section{DOMINIQUE MARGUERIE}

UMR 6566 CReAAH Centre de Recherche en Archéologie, Archéosciences, Histoire (CNRS, Universités de Rennes 1, Rennes 2, Le Mans, Nantes, ministère de la Culture, et INRAP), Laboratoire Archéosciences, Bâtiment 24-25, Université de Rennes 1, Campus de Beaulieu35 042, Rennes cedex, France dominique.marguerie@univ-rennes1.fr

\section{MURIEL FILY}

UMR 6566 CReAAH Centre de Recherche en Archéologie, Archéosciences, Histoire (CNRS, Universités de Rennes 1, Rennes 2, Le Mans, Nantes, ministère de la Culture, et INRAP), Laboratoire Archéosciences, Bâtiment 24-25, Université de Rennes 1, Campus de Beaulieu 35 042, Rennes cedex, France muriel.fily@wanadoo.fr

\section{ANNA BAUDRY}

UMR 6566 CReAAH Centre de Recherche en Archéologie, Archéosciences, Histoire (CNRS, Universités de Rennes 1, Rennes 2, Le Mans, Nantes, ministère de la Culture, et INRAP), Laboratoire Archéosciences, Bâtiment 24-25, Université de Rennes 1, Campus de Beaulieu 35 042, Rennes cedex, France ; INRAP GSO Centre Archéologique de Poitiers, 122, rue de la Bugellerie - 86000 Poitiers, France anna. baudry-dautry@inrap.fr

\section{LAURENT QUESNEL}

UMR 6566 CReAAH Centre de Recherche en Archéologie, Archéosciences, Histoire (CNRS, Universités de Rennes 1, Rennes 2, Le Mans, Nantes, ministère de la Culture, et INRAP), Laboratoire Archéosciences, Bâtiment 24-25, Université de Rennes 1, Campus de Beaulieu 35 042, Rennes cedex, France laurent.quesnel@univ-rennes1.fr

\section{TRISTAN ARBOUSSE-BASTIDE}

7, rue du Pré-Botté - 35000 Rennes 